\title{
Development of Multisensory Neurons and Multisensory Integration in Cat Superior Colliculus
}

\author{
Mark T. Wallace and Barry E. Stein \\ Department of Neurobiology and Anatomy, Bowman Gray School of Medicine of Wake Forest University, Winston-Salem, \\ North Carolina 27157
}

The development of multisensory neurons and multisensory integration was examined in the deep layers of the superior colliculus of kittens ranging in age from 3 to $135 \mathrm{~d}$ postnatal (dpn). Despite the high proportion of multisensory neurons in adult animals, no such neurons were found during the first $10 \mathrm{~d}$ of postnatal life. Rather, all sensory-responsive neurons were unimodal. The first multisensory neurons (somatosensory-auditory) were found at $12 \mathrm{dpn}$, and visually responsive multisensory neurons were not found until 20 dpn. Early multisensory neurons responded weakly to sensory stimuli, had long latencies, large receptive fields, and poorly developed response selectivities. Most surprising, however, was their inability to integrate combinations of sensory cues to produce significant response enhancement (or depression), a characteristic feature of the adult. Responses to combinations of sensory cues differed little from responses to their modality-specific components.

At $28 \mathrm{dpn}$ an abrupt physiological change was noted. Some multisensory neurons now integrated combinations of cross- modality cues and exhibited significant response enhancements when these cues were spatially coincident and response depressions when the cues were spatially disparate. During the next 2 months the incidence of multisensory neurons, and the proportion of these neurons capable of adult-like multisensory integration, gradually increased. Once multisensory integration appeared in a given neuron, its properties changed little with development. Even the youngest integrating neurons showed superadditive enhancements and spatial characteristics of multisensory integration that were indistinguishable from the adult. Nevertheless, neonatal and adult multisensory neurons differed in the manner in which they integrated temporally asynchronous stimuli, a distribution that may reflect the very different behavioral requirements at different ages. The possible maturational role of corticotectal projections in the abrupt gating of multisensory integration is discussed.

Key words: visual; auditory; somatosensory; development; neonate; cross-modality
In contrast to the impressive sensory and sensorimotor capabilities of the adult cat, the newborn kitten seems hopelessly inept. It is unreactive to visual and auditory stimuli, and its motor reactions are sluggish and poorly coordinated (Fox, 1970; Norton, 1974; Van Hof-Van Duin, 1976; Villablanca and Olmstead, 1979; Levine et al., 1980; Sireteanu and Maurer, 1982). At this stage of development the superior colliculus (SC) can play little of its normal role in transforming visual, auditory, and somatosensory stimuli into highly coordinated orientation behaviors. Most SC neurons are unresponsive to natural sensory stimuli, and many loci are not yet capable of evoking overt movements (Stein et al., 1980). Nonetheless, as if to prepare the kitten for its most immediate postnatal task of nuzzling its mother's fur in search of a source of milk, at least some tactile-responsive neurons in the SC become active during late fetal stages. These neurons may play a role in enabling the kitten to find the nipple and get it into its mouth (Stein et al., 1973a; 1980; Larson and Stein, 1984).

A great deal of postnatal maturation is necessary before SC neurons are capable of adult-like responses and the support of mature behaviors. Auditory responses do not appear until late in the first postnatal week, and deep layer visual responses are not

\footnotetext{
Received Oct. 17, 1996; revised Jan. 9, 1997; accepted Jan. 15, 1997.

This work was supported by National Institutes of Health Grant EY06562. We thank Nancy London for her assistance in all phases of this project.

Correspondence should be addressed to Dr. Mark T. Wallace, Department of Neurobiology and Anatomy, Bowman Gray School of Medicine/Wake Forest University, Medical Center Boulevard, Winston-Salem, NC 27157-1010.

Copyright (C) 1997 Society for Neuroscience $0270-6474 / 97 / 172429-16 \$ 05.00 / 0$
}

seen until approximately 3 weeks postnatal (visual neurons in the purely visual superficial layers are active earlier) (Stein et al., 1973a; Wallace et al., 1993b; Kao et al., 1994b). The responses of early SC neurons are quite different from those of the adult: they are weak, have long latencies, and lack most of the selectivity seen in the adult (Stein et al., 1973a,b). By the second postnatal month, however, such responses are more vigorous, rapid, and selective, and overt orienting movements can be evoked by electrical stimulation of the SC. By this time the kitten has developed a great deal of motor coordination. Succeeding developmental stages produce far smaller changes as the kitten's sensory and motor skills are further sharpened.

Although a good deal is known about the developmental changes that take place during this period in terms of the unimodal properties of SC neurons (Stein et al., 1993), little is known about the development of perhaps the most characteristic feature of these neurons in the adult: their ability to integrate inputs from multiple sensory modalities. It is this ability that gives the SC its central role in mediating cross-modality orientation behaviors.

In the adult cat, visual, auditory, and somatosensory inputs converge on individual SC neurons, thus rendering two-thirds or more of them "multisensory" (Stein et al., 1976; Wallace et al., 1993a). By synthesizing these convergent inputs, multisensory SC neurons give rise to responses that are significantly different from those that are predicted on the basis of a simple summation of these inputs (Meredith and Stein, 1986a,b). Depending on the spatial and temporal relationships among the stimuli, dramatic 
response enhancements or depressions can be produced (Stein and Meredith, 1993). Because multisensory neurons are a major constituent of the output pathways to the brainstem and spinal cord that influence overt behaviors (Meredith et al., 1992; Wallace et al., 1993a), the same principles that govern their cross-modal neural interactions are applicable to the animal's behavior (Stein et al., 1989; Wilkinson et al., 1996).

The present experiments were initiated to examine the ontogeny of these cross-modality integrative capabilities. For despite the importance of achieving mature unimodal response properties, these properties provide only a limited perspective regarding the information-processing capabilities of this structure. Until SC neurons are capable of integrating information from different sensory modalities, the advantage of having multiple sensory inputs converging on individual SC neurons is not realized.

A portion of this work has been published previously in abstract form (Wallace et al., 1993b).

\section{MATERIALS AND METHODS}

All procedures were performed in compliance with the Guide for the Care and Use of Laboratory Animals (National Institutes of Health Publication No. 91-3207) at The Bowman Gray School of Medicine, which is accredited by the American Association for Accreditation of Laboratory Animal Care. Details of surgery, stimulation, and data analyses are similar to those used previously in adult animals, and further methodological detail can be obtained from Wallace et al. (1993a) and Wallace and Stein (1994).

\section{General surgical procedures}

Kittens 3-135 d postnatal (dpn) were anesthetized with a combination of ketamine hydrochloride $(5-25 \mathrm{mg} / \mathrm{kg}$, i.m.) and acepromazine maleate $(0.2-0.4 \mathrm{mg} / \mathrm{kg}$, i.m.). Surgical anesthesia was maintained with halothane $(1.0-4.0 \%)$, and each animal was artificially ventilated. A craniotomy was made over visual cortex to allow access to the SC. A head-holder was attached to the cranium with screws and dental acrylic to hold the animal during recording without obstructing the face and ears. The saphenous vein was cannulated for the delivery of intravenous fluids (lactated Ringer's solution, $1-4 \mathrm{cc} / \mathrm{hr}$ ), and paralysis was induced (pancuronium bromide, $2 \mathrm{mg} \cdot \mathrm{kg}^{-1} \cdot \mathrm{hr}^{-1}$, i.v.). Anesthesia was maintained during the recording session with ketamine hydrochloride $\left(4-8 \mathrm{mg} \cdot \mathrm{kg}^{-1} \cdot \mathrm{hr}^{-1}\right)$, expiratory $\mathrm{CO}_{2}$ was maintained between 3.8 and $4.5 \%$, and body temperature was kept at $37-38^{\circ} \mathrm{C}$ with a circulating hot water pad. Periodic recovery from paralysis was used as a measure of adequate depth of anesthesia. The pupils were dilated with $1 \%$ atropine sulfate, and the eyes were fitted with contact lenses to prevent corneal drying and to correct for refractive errors. When the ocular media allowed for reverse ophthalmoscopy, the optic disks were projected onto a translucent $91-\mathrm{cm}$ diameter Plexiglas hemisphere.

\section{Recording}

Parylene-insulated tungsten electrodes (impedance 2-5 M $\Omega$ ) were lowered to the surface of the SC and then advanced using a hydraulic microdrive. Single- and multiunit neural activity was recorded and amplified, and discharges were routed to an oscilloscope, audio monitor, and computer for subsequent off-line analyses.

\section{Stimulus delivery/recording procedures}

Each neuron that was encountered was evaluated. To avoid the possibility of missing neurons with little or no spontaneous activity, the electrode was advanced in small $(10-15 \mu \mathrm{m})$ steps, and visual, auditory, and somatosensory search stimuli were presented. Visual search stimuli consisted of moving and flashed spots and bars (luminance: $20-100 \mathrm{~cd} / \mathrm{m}^{2}$ against a background of $3 \mathrm{~cd} / \mathrm{m}^{2}$ ) projected onto the Plexiglas hemisphere. Receptive field borders were mapped manually on the hemisphere with a pantoscope and transferred to a standardized representation of visual space. Auditory search stimuli consisted of clicks, whistles, and broad band (i.e., white and pink) noise bursts [duration: 50-200 msec; intensity: $40-100 \mathrm{~dB}$ sound pressure level (SPL)]. The noise bursts were delivered from either (or both) of a pair of moveable hoop-mounted speakers, which were also used to map receptive fields. Somatosensory search stimuli consisted of manual deflections of the hair with a camel- hair brush, vertical indentations of the skin with a small probe or von Frey hair, deep subcutaneous pressure, lateral skin stretch, calibrated forceps pinch, and rotation of the joints. The minimum stimulus necessary to evoke a response was used to map the receptive field of the neuron. After the initial modality classification and assessment of response specificity, each neuron was subjected to a more quantitative series of tests. To determine mean response latency, 8-10 repetitions of identical computer-controlled stimuli were delivered, and the mean time to the first impulse was determined.

Multisensory definitions/tests/analyses. A multisensory neuron was defined as one that responded to cues from more than one sensory modality or whose responses to one modality were significantly altered by the presence of a stimulus from another modality (Stein and Meredith, 1993). Once the modality selectivity (e.g., visual-somatosensory) of a multisensory neuron was established, its responses to each single-modality stimulus (e.g., visual alone, somatosensory alone) and multisensory combination (e.g., visual-somatosensory) were evaluated quantitatively using reproducible, electronically controlled stimuli initiated by a TTL pulse from a 486PC computer.

Visual stimuli were generated from a high-intensity Prado projector fitted with a series of diaphragms to change the size and shape of the bar and spot stimuli presented (for further detail, see Meredith and Stein, 1986a). A given stimulus was moved across the receptive field in a series of different directions and velocities. An electronic shutter in the light path allowed the same stimuli to be flashed on and off at specific positions in the receptive field and at different iterative rates. Auditory stimuli were presented via hoop-mounted speakers as described above, and somatosensory stimuli were delivered using an electronically controlled moving coil vibrator/shaker (Ling 502A). One of a series of probe tips or brushes was loaded against the hair or skin, and the amplitude and velocity of the stimulus were computer-controlled (Clemo and Stein, 1986).

Each unimodal stimulus [e.g., visual (V), somatosensory (S)], and stimulus combination (VS) was presented 8-16 times at 10-30 sec interstimulus intervals with the different conditions (V, S, VS) randomly interleaved. A multisensory interaction was operationally defined as a significant (two-tailed $t$ test; $p<0.05$ ) increase or decrease in the number of impulses to the combined stimuli compared with the most effective single-modality stimulus. The magnitude of this multisensory response enhancement or depression was calculated as:

$$
\left(C M-S M_{\max }\right) /\left(S M_{\max }\right) \times 100=\% \text { interaction, }
$$

where $C M$ is the mean number of impulses evoked by the combinedmodality stimulus and $S M_{\max }$ is the mean number of impulses evoked by the most effective single-modality stimulus.

Histology, euthanasia, and cluster analyses. For each electrode penetration the depth of every recorded neuron was noted, and electrolytic lesions were made at several locations. At the end of the experiment the animal was euthanized with an overdose of sodium pentobarbital (100

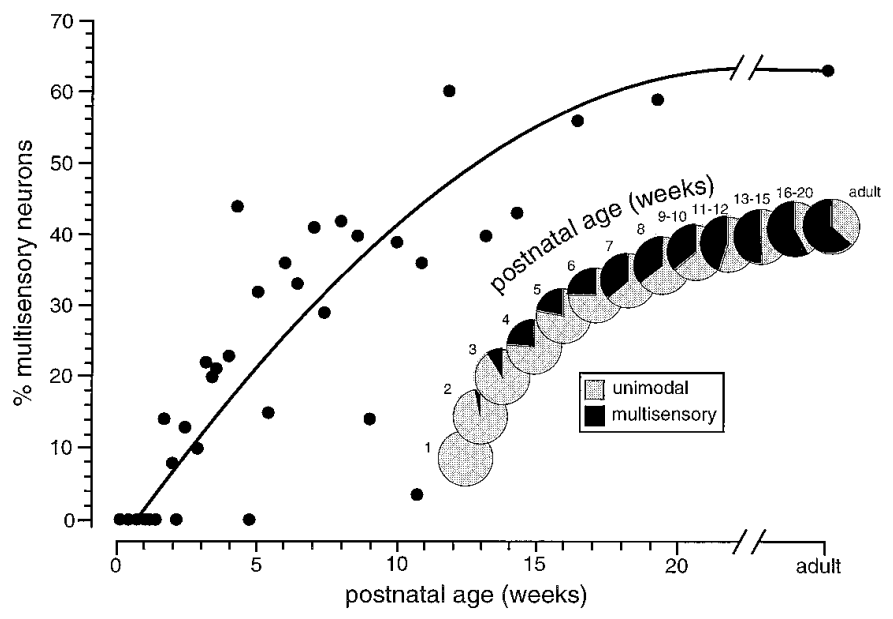

Figure 1. The developmental chronology of multisensory neurons. The percentage of multisensory neurons in the deep layer sensory-responsive population is plotted as a function of postnatal age. Pie charts in the inset show the expansion of the multisensory population as development progresses. 


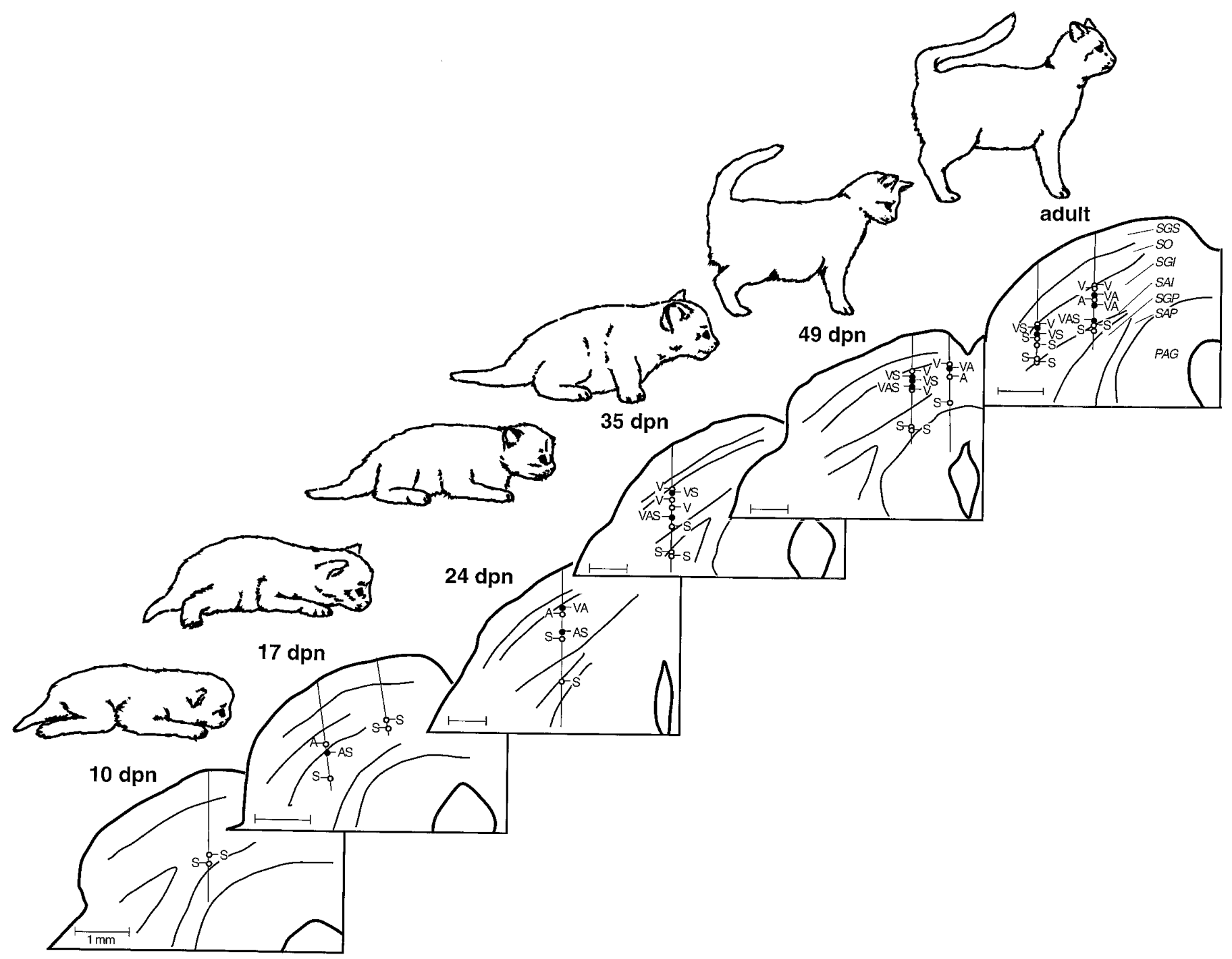

Figure 2. The incidence of sensory-responsive neurons (i.e., unimodal and multisensory) in the deep SC increases with increasing postnatal age. Shown are representative histological reconstructions of coronal sections through the SC mapping the location of unimodal and multisensory neurons in individual animals at six developmental stages. Vertical lines on each section represent electrode penetrations, and the location of each recorded sensory-responsive neuron is depicted with a circle (open circles = unimodal neurons; closed circles = multisensory neurons; $V$, visual; $A$, auditory; $S$, somatosensory; $V A$, visual-auditory, etc.). Scale bar, $1 \mathrm{~mm}$. $S G S$, Stratum griseum superficiale; $S O$, stratum opticum; $S G I$, stratum griseum intermediale; $S A I$, stratum album intermediale; $S G P$, stratum griseum profundum; $S A P$, stratum album profundum, $P A G$, periaqueductal gray.

$\mathrm{mg} / \mathrm{kg}$, i.v.) and perfused transcardially with saline followed by formalin. The midbrain was blocked stereotaxically, removed, and placed in sucrose overnight. Frozen sections (50 $\mu \mathrm{m}$ thickness) were taken in the coronal plane and were counterstained with neutral red to facilitate the distinction of laminar borders. The outline of the tissue, along with laminar boundaries and the positions of electrode tracts and lesions, was traced using a projection microscope and then input into a Macintosh PowerPC by means of a digitizing pad. After tissue shrinkage was accounted for, neuronal locations were plotted onto these representations. In addition, these distributions were examined for clustering by modality type. Cluster analyses were performed in which the probability that neighboring neurons were of like modality or modalities was assessed using $\chi^{2}$ testing. In this form of analysis, neurons were simply listed in the order in which they were encountered, with no weight given to the distance between two adjacent neurons (Wallace et al., 1996).

\section{RESULTS}

Data were collected from a total of 665 neurons in the deep layers (below stratum opticum) of the SC in 44 animals. A total of 458 of these neurons were obtained from 41 animals at various devel- opmental stages from 3-135 dpn. Data from 207 neurons in three adults were used for comparison (Table 1).

\section{Developmental chronology}

Few active neurons were encountered in the SC of the youngest (i.e., 3-5 dpn) animals studied, and none of these neurons were multisensory (Table 1). Many long electrode traverses were made in which neither spontaneous nor sensory-evoked activity was encountered. Nevertheless, periodically a neuron responsive to sensory stimuli was found. Invariably in these very young animals such a neuron was activated exclusively by somatosensory stimuli. The earliest auditory-responsive neuron was found at $5 \mathrm{dpn}$, and it too was unimodal. The first neuron influenced by more than a single sensory modality, and thus by definition the first "multisensory" neuron, was encountered at $12 \mathrm{dpn}$ (Table 1). This neuron, as well as multisensory neurons found at 14 and $17 \mathrm{dpn}$, was responsive to somatosensory and auditory cues, reflecting the two 


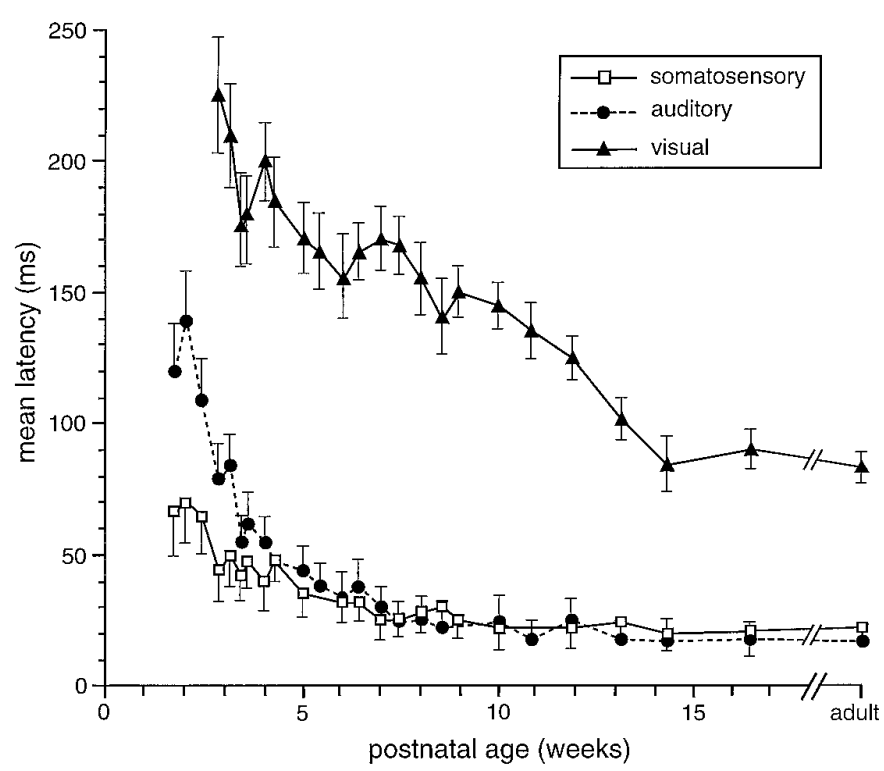

Figure 3. The development of unimodal response latencies in multisensory neurons. Errors bars represent SEM.

functional modalities in the deep SC at this time. It was not until $20 \mathrm{dpn}$ that the first deep-layer neurons responsive to visual stimuli (both unimodal and multisensory) were found. Early sensory-responsive neurons had very large receptive fields, responded with few impulses to each stimulus presentation, had long latencies, and habituated rapidly to the delivery of repeated stimuli.

During the next 3 months, the incidence of multisensory neurons increased substantially, with such neurons becoming a progressively larger proportion of the sensory-responsive population (Fig. 1). By 6 postnatal weeks multisensory neurons represented $\sim 25 \%$ of the deep layer sensory population, and by 13 postnatal weeks they comprised about half of this population. Their numbers continued to rise slowly after this, reaching adult-like values (i.e., $\sim 63 \%$ ) after $\sim 4$ months.

The distribution of the different multisensory neuron types changed with development. In large measure this shift was the result of the delayed appearance and then gradual increase of deep layer visual responses. When they first appeared at $20 \mathrm{dpn}$, visually responsive multisensory neurons represented a small component $(5 \%)$ of the sensory-responsive population. Gradually, the incidence of visual-somatosensory, visual-auditory, and trimodal neurons increased, with a corresponding decline in the proportion of auditory-somatosensory neurons (Table 1). By late postnatal ages (100-135 dpn), neurons with a visual component dominated the multisensory population, having an incidence in excess of $95 \%$.

\section{Laminar location}

Multisensory neurons were never found in the superficial SC layers, which remained exclusively visual throughout development. Typically, multisensory neurons were first encountered in the uppermost reaches of the intermediate layers, in stratum griseum intermediale (SGI) (Fig. 2), where they were most abundant at all developmental stages. Below the SGI, the incidence of multisensory neurons declined, a feature that was also true at all developmental ages. As they became a significant component of the sensory-responsive SC population, multisensory neurons were found to be grouped in vertical clusters. These clusters, which were retained into adulthood, spanned 100-500 $\mu \mathrm{m}$ and were first evident at $42 \mathrm{dpn}$, when multisensory neurons constituted slightly more than $25 \%$ of the deep SC population. Cluster analyses at this and all subsequent developmental stages revealed that a multisensory neuron was significantly more likely ( $\chi^{2}$ analysis, $p<0.05$ ) to be the neighbor of another multisensory neuron than to be found next to a unimodal neuron. Regional analyses of the SC at various ages failed to reveal gradients in the development of multisensory neurons along the various SC axes. Thus, even in very young animals, multisensory neurons were as likely to be found rostral or medial as they were to be found caudal or lateral.

\section{Response latencies}

Latencies to optimally effective sensory stimuli were examined in each neuron encountered. In the earliest multisensory neurons, response latencies were substantially longer than in older animals.

Table 1. The changing distribution of multisensory and unimodal neurons in the deep layers of the SC during development

\begin{tabular}{lrrrrr} 
& \multicolumn{3}{c}{ Multisensory neurons } & Unimodal \\
\cline { 2 - 4 } Age in dpn & VA & VS & VAS & AS & neurons \\
\hline $3(n=1)$ & 0 & 0 & 0 & 0 & 2 \\
$5(1)$ & 0 & 0 & 0 & 0 & 7 \\
$7(2)$ & 0 & 0 & 0 & 0 & 8 \\
$8(1)$ & 0 & 0 & 0 & 0 & 3 \\
$10(2)$ & 0 & 0 & 0 & 0 & 11 \\
$12(1)$ & 0 & 0 & 0 & 1 & 6 \\
$14(2)$ & 0 & 0 & 0 & 1 & 12 \\
$15(1)$ & 0 & 0 & 0 & 0 & 6 \\
$17(1)$ & 0 & 0 & 0 & 2 & 13 \\
$20(2)$ & 0 & 1 & 0 & 1 & 18 \\
$22(1)$ & 1 & 1 & 0 & 0 & 7 \\
$24(2)$ & 1 & 2 & 2 & 1 & 24 \\
$25(1)$ & 1 & 2 & 0 & 0 & 11 \\
$28(2)$ & 2 & 2 & 1 & 0 & 17 \\
$30(1)$ & 2 & 1 & 1 & 0 & 5 \\
$33(1)$ & 0 & 0 & 0 & 0 & 10 \\
$35(1)$ & 2 & 3 & 1 & 0 & 13 \\
$38(1)$ & 2 & 0 & 0 & 0 & 11 \\
$42(2)$ & 8 & 4 & 2 & 1 & 27 \\
$45(1)$ & 0 & 0 & 1 & 0 & 2 \\
$49(1)$ & 4 & 4 & 1 & 0 & 13 \\
$52(2)$ & 5 & 1 & 1 & 0 & 17 \\
$56(1)$ & 0 & 3 & 2 & 0 & 7 \\
$60(1)$ & 5 & 2 & 1 & 0 & 12 \\
$63(1)$ & 0 & 1 & 0 & 0 & 6 \\
$70(1)$ & 1 & 4 & 2 & 0 & 11 \\
$76(1)$ & 4 & 0 & 0 & 0 & 7 \\
$83(1)$ & 3 & 5 & 1 & 0 & 6 \\
$92(1)$ & 5 & 0 & 1 & 0 & 9 \\
$100(1)$ & 4 & 3 & 2 & 0 & 12 \\
$115(1)$ & 1 & 3 & 0 & 1 & 4 \\
$135(1)$ & 6 & 3 & 1 & 0 & 7 \\
Kitten $(41)$ & 57 & 45 & 20 & 8 & 328 \\
Adult (3) & 57 & 50 & 19 & 4 & 77 \\
& & & & &
\end{tabular}

Grand total

Totals $(n=44) \quad 114 \quad 95 \quad 39 \quad 12 \quad 405 \quad$ neurons $=665$

VA, Visual-auditory; VS, visual-somatosensory; VAS, visual-auditory-somatosensory; AS, auditory-somatosensory. 


\begin{tabular}{|c|c|c|c|c|c|c|c|c|c|c|c|}
\hline \multirow{2}{*}{$\begin{array}{l}\text { Postnatal age } \\
\text { (weeks) }\end{array}$} & \multicolumn{4}{|c|}{ Velocity preference } & \multicolumn{4}{|c|}{ Receptor type } & \multicolumn{3}{|c|}{ Adaptation } \\
\hline & $n$ & High (\%) & Low $(\%)$ & None $(\%)$ & $n$ & Hair (\%) & Skin $(\%)$ & Deep $(\%)$ & $n$ & Fast $(\%)$ & Slow $(\%)$ \\
\hline 2 & 1 & 0 & 0 & 100 & 1 & 0 & 100 & 0 & 0 & & \\
\hline 3 & 4 & 50 & 0 & 50 & 4 & 75 & 25 & 0 & 3 & 67 & 33 \\
\hline 4 & 7 & 71 & 0 & 29 & 7 & 71 & 29 & 0 & 6 & 100 & 0 \\
\hline 5 & 5 & 100 & 0 & 0 & 4 & 75 & 25 & 0 & 4 & 100 & 0 \\
\hline 6 & 4 & 75 & 0 & 25 & 4 & 100 & 0 & 0 & 4 & 100 & 0 \\
\hline 7 & 8 & 75 & 13 & 13 & 7 & 71 & 14 & 14 & 7 & 86 & 14 \\
\hline 8 & 6 & 83 & 0 & 17 & 6 & 83 & 17 & 0 & 5 & 100 & 0 \\
\hline 9 & 5 & 100 & 0 & 0 & 6 & 100 & 0 & 0 & 5 & 100 & 0 \\
\hline 10 & 0 & & & & 1 & 100 & 0 & 0 & 0 & & \\
\hline 11 & 5 & 80 & 0 & 20 & 4 & 100 & 0 & 0 & 5 & 100 & 0 \\
\hline 12 & 5 & 100 & 0 & 0 & 6 & 83 & 17 & 0 & 4 & 100 & 0 \\
\hline 14 & 1 & 100 & 0 & 0 & 1 & 100 & 0 & 0 & 1 & 100 & 0 \\
\hline 15 & 4 & 100 & 0 & 0 & 4 & 75 & 0 & 25 & 3 & 67 & 33 \\
\hline 17 & 3 & 100 & 0 & 0 & 4 & 100 & 0 & 0 & 3 & 100 & 0 \\
\hline 20 & 4 & 75 & 0 & 25 & 3 & 67 & 33 & 0 & 4 & 100 & 0 \\
\hline Adult & 63 & 83 & 5 & 12 & 66 & 76 & 23 & 2 & 59 & 97 & 3 \\
\hline
\end{tabular}

As an example, an auditory-somatosensory neuron isolated in a $14 \mathrm{dpn}$ animal had a mean auditory latency of $140 \mathrm{msec}$ and a mean somatosensory latency of $71 \mathrm{msec}$. This contrasts strikingly with the mean latencies for these responses in adults, which are 16 and $23 \mathrm{msec}$, respectively. Similarly, the mean visual latency in the earliest visually responsive multisensory neuron encountered (20 dpn) was $224 \mathrm{msec}$, nearly three times the adult mean of $82 \mathrm{msec}$. For each modality there was a similar developmental trend in response latency: a precipitous decline during the week after the initial appearance of sensory responses followed by a more gradual decline until adult values were achieved (Fig. 3). Although auditory and somatosensory responses had adult-like latencies by the middle of the second postnatal month, visual latencies did not approach their mature values until 3 postnatal months. The comparatively slow maturation of deep layer visual responses was evident for a number of the functional properties examined here (see below).

\section{Receptive fields}

Early multisensory neurons were characterized by large receptive fields. Most impressive in this regard were auditory receptive fields (Fig. 4). Although visual receptive fields in very young animals often covered a substantial portion of the contralateral hemifield, and somatosensory receptive fields covered much, if not all, of the contralateral body (and in a few cases the entire body), early auditory responses were omnidirectional: they could be evoked from anywhere in auditory space. Within a week of their appearance, a number of auditory-responsive neurons had developed true receptive fields, although these tended to be exceedingly large. For each of the modalities, the developmental trend for receptive field size was similar in form to that seen for response latency (Fig. 3): an initial rapid decline followed by a gradual decrement until the adult values were achieved at $\sim 12$ weeks (Fig. 4).

The close parallels among the modalities provided no evidence that changes in receptive field size in one modality had to precede this change in another modality to provide spatial restriction cues (see Discussion). Furthermore, during this period of receptive field contraction, the different modality-specific receptive fields of a given multisensory neuron maintained a good spatial correspondence. This correspondence became increasingly evident as receptive fields became smaller (Fig. 4).

\section{Modality-specific response properties of multisensory neurons}

\section{Somatosensory}

Somatosensory responses in multisensory neurons underwent less impressive developmental changes than did auditory and visual responses. Although in the earliest examples somatosensory receptive fields were large, responses were sluggish, and latencies were long (see above), these neurons exhibited many of the characteristic properties seen in older animals: they adapted rapidly to maintained stimulation, were largely cutaneous, and preferred high velocity stimuli (Table 2).

\section{Auditory}

The use of a free-field paradigm for delivering auditory stimuli made it difficult to rigorously assess binaural categories (Goldberg and Brown, 1969). Nevertheless, the transition from omnidirectional responses to discrete contralateral receptive fields seemed to be driven in large measure by the development of interactions between inputs from the two ears. Thus, the first omnidirectional auditory-responsive multisensory neurons responded equally well to contralateral, ipsilateral, and synchronous bilateral free-field stimuli and were classified as "CI" (responsive to contralateral and ipsilateral stimuli) (Fig. 5). During the third postnatal week, neurons were found in which bilateral stimulation resulted in enhanced responses; such neurons were categorized "CI/E" (responsive to contralateral and ipsilateral stimuli, and enhanced by their combination). In the fourth postnatal week neurons appeared that could not be excited by ipsilateral stimuli. By virtue of their lack of ipsilateral response, these neurons (classified as "C") were the first to exhibit circumscribed receptive fields.

It was not until the fifth postnatal week that neurons appeared which exhibited clear inhibitory influences. The first of these were excited by a contralateral stimulus, failed to respond to an ipsilateral stimulus, and exhibited a depressed response to the combination of contralateral and ipsilateral stimuli. Such "C/D" neurons typically 

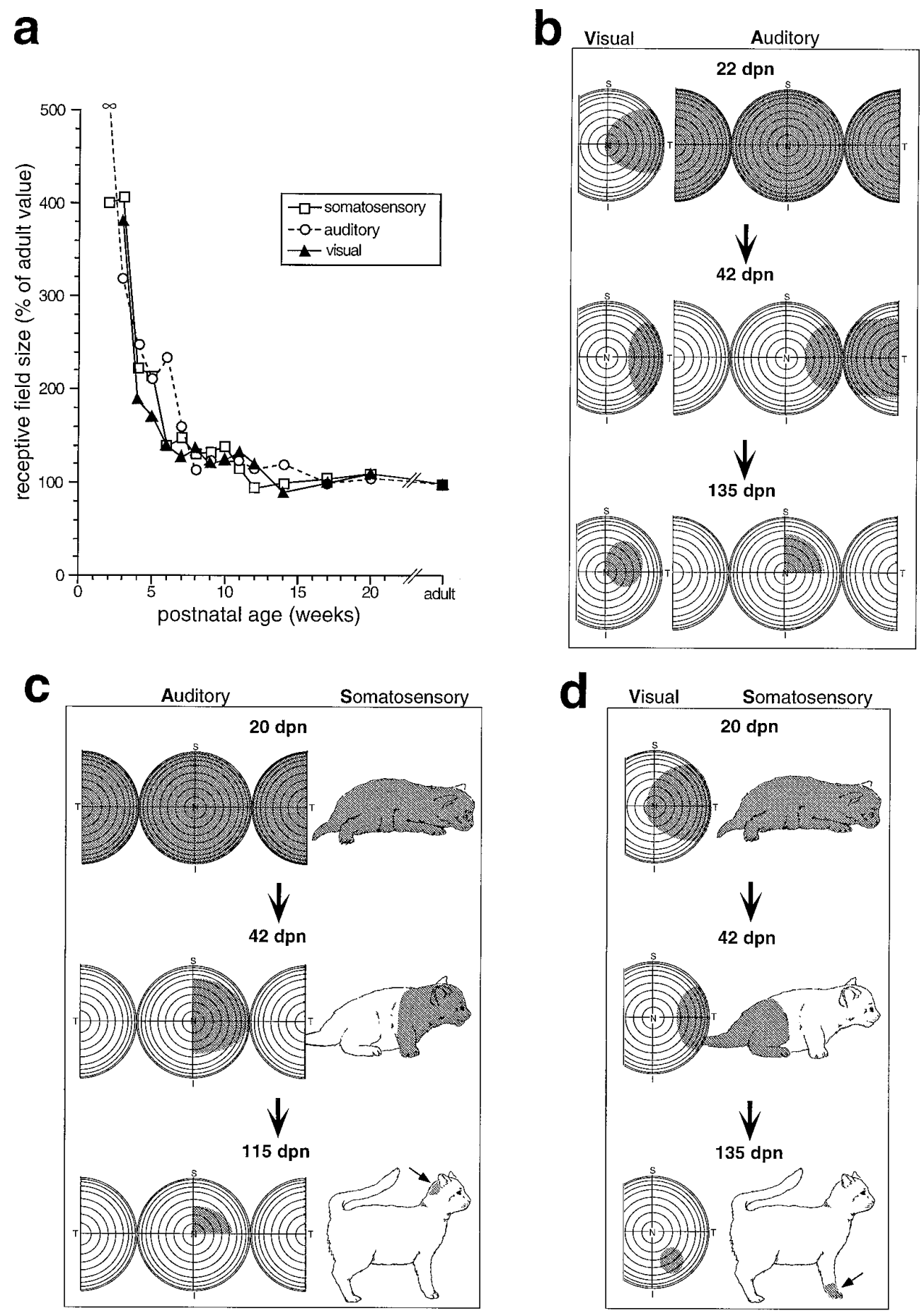

Figure 4. The receptive fields of multisensory neurons decline substantially in size during development. $a$, Receptive field size (as a percentage of the mean adult value) is plotted as a function of postnatal age. Note the rapid decline for each modality-specific receptive field during the first $4-6$ weeks. $b$, Representative receptive fields (shading) of visual-auditory neurons at three ages $(22,42$, and 135 dpn) are plotted onto representations of visual and auditory space. Frontal auditory space is represented on the central hemisphere, and caudal space is represented by a hemisphere that has been split and both halves have been folded forward. Each concentric circle represents $10^{\circ}$. $c$, Representative receptive fields of auditory-somatosensory neurons at three ages $(20,42$, and $115 \mathrm{dpn})$. An arrow points to the small somatosensory receptive field at $115 \mathrm{dpn}$. $d$, Representative receptive fields of visualsomatosensory neurons at three ages (20,42, and $135 \mathrm{dpn})$. An arrow points to the small somatosensory receptive field at $135 \mathrm{dpn}$. $n$, Nasal; $T$, temporal; $S$, superior; $I$, inferior.

had the smallest receptive fields, often having only $45-60^{\circ}$ of azimuthal extent. The incidence of $\mathrm{C} / \mathrm{D}$ neurons increased rapidly after their initial appearance and was paralleled by a concomitant decline in the proportion of $\mathrm{CI}$ and $\mathrm{CI} / \mathrm{E}$ neurons. By 8 weeks postnatal,
C/D neurons represented a relatively large and stable proportion $(50-70 \%)$ of the auditory-responsive population.

During development, mean auditory thresholds declined, from an average of $71 \mathrm{~dB}$ SPL (range, 61-98 dB SPL) at 3 postnatal 


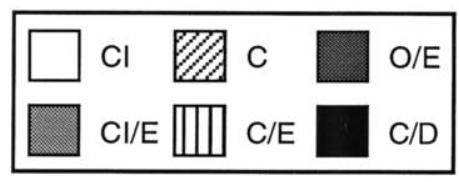

(5)

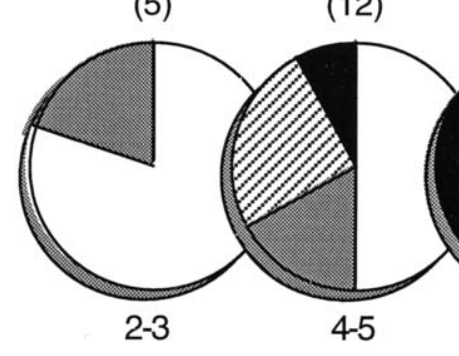

(17)
(7)

(16)
(9)
(35)

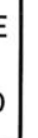

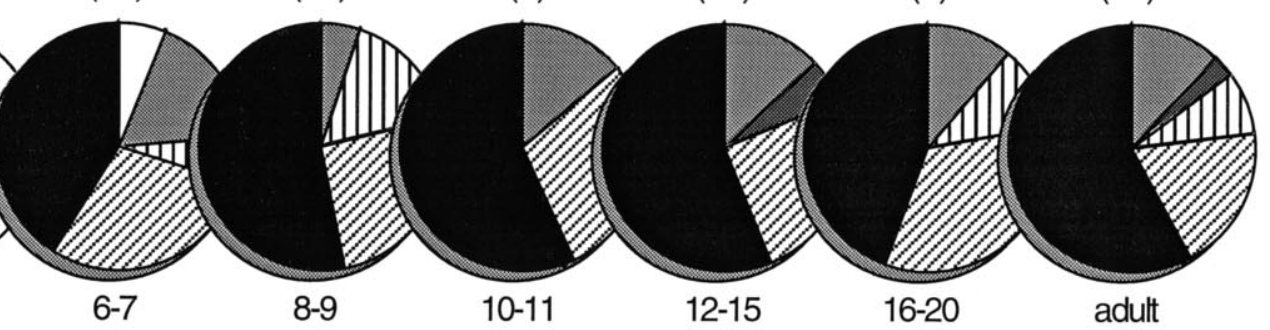

postnatal age (weeks)

Figure 5. The development of auditory response categories in multisensory neurons. Initially, auditory responses can be elicited from multisensory neurons by stimuli positioned in both contralateral and ipsilateral space. These "omnidirectional" neurons respond to the pairing of stimuli from both sides, with either no interaction $(C I)$ or an enhanced response $(C I / E)$. During development, other response categories appear that reflect the appearance of more discrete excitatory receptive fields (see text). $C$, Response to contralateral stimulus; $I$, response to ipsilateral stimulus; $O$, no response; $E$, enhanced response to contralateral-ipsilateral pairing; $D$, depressed response to contralateral-ipsilateral pairing. Numbers in parentheses above pie charts represent the number of neurons in each postnatal age grouping.

weeks to $58 \mathrm{~dB}$ SPL (range, 45-76 dB SPL) at 6 postnatal weeks, and finally to the adult value of $53 \mathrm{~dB}$ at 13 postnatal weeks (range, 41-70 dB SPL).

\section{Visual}

The earliest visually responsive multisensory neurons exhibited little of the stimulus selectivity found in adults. Typically, they responded equally well to stationary flashed or moving stimuli and seemed not to code the features of the stimulus or the parameters of the movement. For example, the first neuron exhibiting direction selectivity was not encountered until $28 \mathrm{dpn}$. After this time, the incidence of directionally selective multisensory neurons increased gradually to the adult-like level of $40 \%$ at $\sim 4$ months of age. The developmental changes (or constancies) in velocity se- lectivity, binocularity, and within-field spatial summation and inhibition as well as surround inhibition are shown in Table 3.

\section{Multisensory integration}

Although multisensory neurons in animals younger than 28 dpn responded reliably, and sometimes robustly, to inputs from more than a single sensory modality, they were distinctly different from multisensory neurons in the adult. Regardless of the nature of the multisensory neuron (e.g., auditory-somatosensory, visual-auditory, etc.), or how the physical relationships and parameters of the stimuli were manipulated (timing, spatial location, size, etc.), responses to combinations of different sensory stimuli were generally neither better nor worse than their responses to the best unimodal stimulus when presented alone. This is illustrated by the

\begin{tabular}{|c|c|c|c|c|c|c|c|}
\hline $\begin{array}{l}\text { Postnatal } \\
\text { age } \\
\text { (weeks) }\end{array}$ & $\begin{array}{l}\text { Direction } \\
\text { selectivity } \\
\%(n)\end{array}$ & $\begin{array}{l}\text { Response } \\
\text { to flash } \\
\%(n)\end{array}$ & $\begin{array}{l}\text { Surround } \\
\text { inhibition } \\
\%(n)\end{array}$ & $\begin{array}{l}\text { Spatial } \\
\text { summation } \\
\%(n)\end{array}$ & $\begin{array}{l}\text { Spatial } \\
\text { inhibition } \\
\%(n)\end{array}$ & $\begin{array}{l}\text { Velocity } \\
\text { selectivity } \\
\%(n)\end{array}$ & $\begin{array}{l}\text { Habituation } \\
\%(n)\end{array}$ \\
\hline 3 & $0(1)$ & $100(1)$ & $0(1)$ & $100(1)$ & $0(1)$ & $0(1)$ & $100(1)$ \\
\hline 4 & $0(7)$ & $88(8)$ & $20(5)$ & $86(7)$ & $33(6)$ & $0(5)$ & $100(4)$ \\
\hline 5 & $0(5)$ & $86(7)$ & $0(4)$ & $67(6)$ & $67(6)$ & $20(5)$ & $100(4)$ \\
\hline 6 & $0(4)$ & $100(6)$ & $0(3)$ & $100(5)$ & $75(4)$ & $20(5)$ & $100(5)$ \\
\hline 7 & $18(11)$ & 71 (14) & $22(9)$ & $58(12)$ & $50(10)$ & $29(7)$ & 73 (11) \\
\hline 8 & $11(9)$ & $67(12)$ & $0(6)$ & 64 (11) & 45 (11) & $44(9)$ & 64 (11) \\
\hline 9 & $29(7)$ & $40(10)$ & $20(5)$ & $56(9)$ & $33(9)$ & $50(8)$ & $71(7)$ \\
\hline 10 & $0(1)$ & $100(1)$ & (0) & $0(1)$ & $0(1)$ & (0) & (0) \\
\hline 11 & $29(7)$ & $55(9)$ & $20(5)$ & $50(8)$ & $43(7)$ & $50(6)$ & $50(6)$ \\
\hline 12 & $33(3)$ & $50(6)$ & $0(4)$ & $25(4)$ & $0(4)$ & $67(6)$ & $43(7)$ \\
\hline 14 & $0(3)$ & $40(5)$ & $25(4)$ & $33(3)$ & $0(3)$ & $50(4)$ & $25(8)$ \\
\hline 15 & $40(5)$ & $43(7)$ & $0(6)$ & $50(4)$ & $33(3)$ & $75(4)$ & $33(6)$ \\
\hline 17 & $0(2)$ & $33(3)$ & $0(3)$ & $33(3)$ & $0(3)$ & $67(3)$ & $0(3)$ \\
\hline 20 & $50(6)$ & $33(6)$ & $17(5)$ & $38(8)$ & $43(7)$ & $86(7)$ & $17(6)$ \\
\hline Adult & $34(70)$ & 39 (105) & $16(56)$ & $45(95)$ & $30(92)$ & $74(69)$ & $27(74)$ \\
\hline
\end{tabular}




\section{Visual}
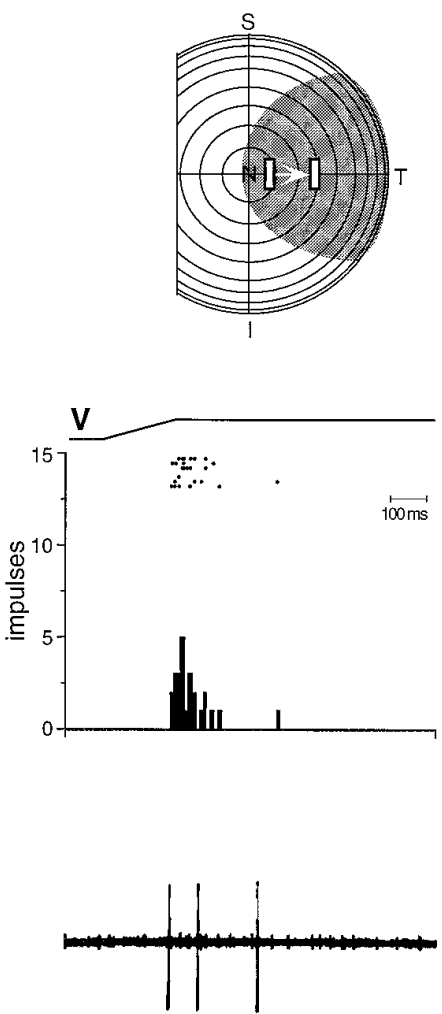

\section{Somatosensory}
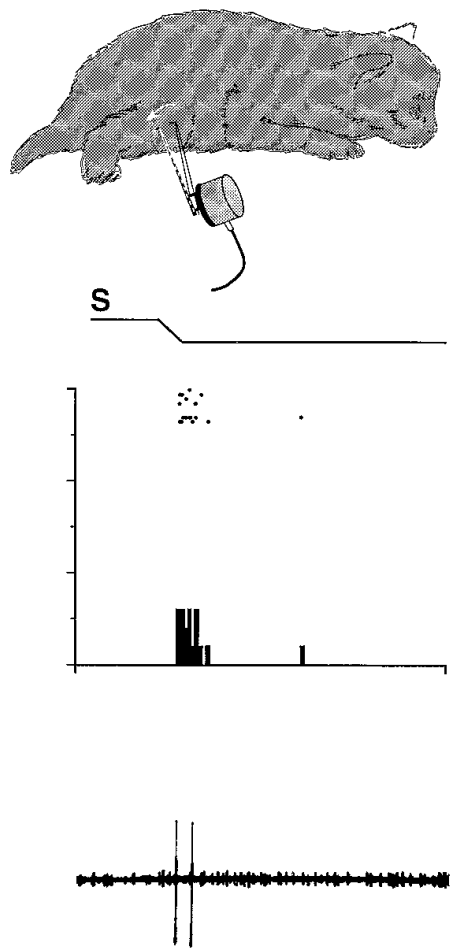

\section{Visual + Somatosensory}
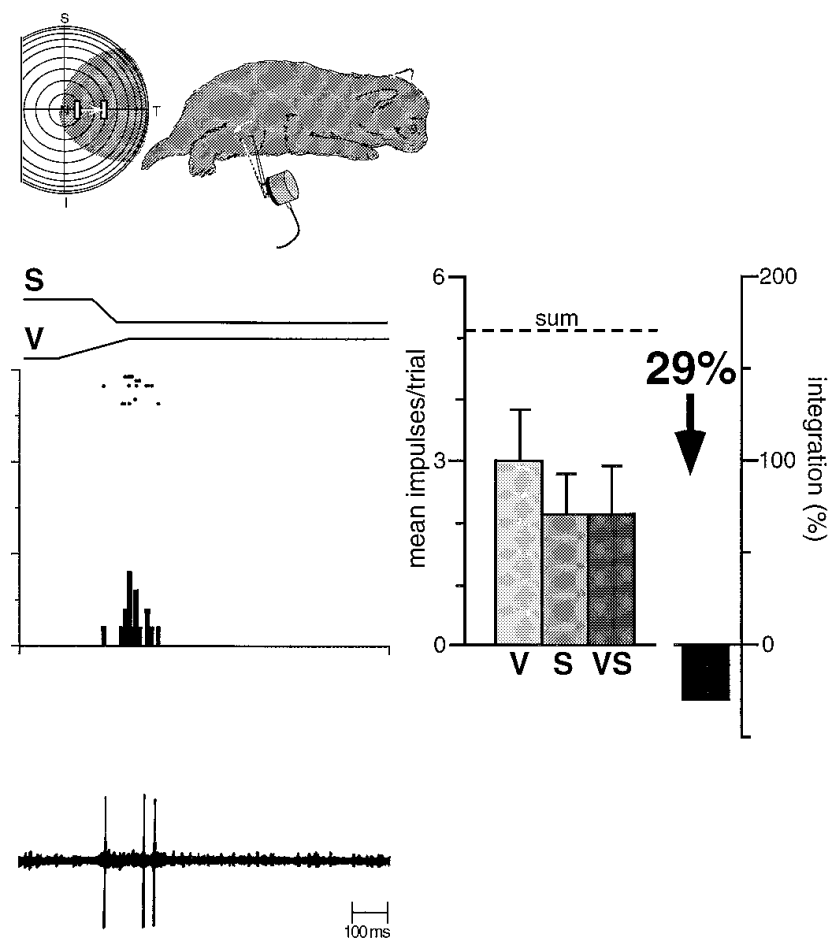

Figure 6. The earliest multisensory neurons do not integrate cross-modality sensory cues to produce the response changes that characterize adults. This figure illustrates the responses of a visual-somatosensory neuron in a $20 \mathrm{dpn}$ animal. Top, Visual and somatosensory receptive fields are depicted by shading and are shown for each of the three stimulus conditions. The visual stimulus is a bar of light moving in the direction and amplitude depicted by the bars and arrow within the receptive field. The somatosensory stimulus is a probe mounted onto a lever that deflects hairs and skin within the receptive field (probe movement depicted by arrow). Middle, Rasters, peristimulus histograms, and bar graphs illustrate the responses of this neuron to the unimodal and multisensory stimulus conditions. The electronic trace driving the stimulus ( $V$, visual; $S$, somatosensory) is shown above the rasters. Each dot of the raster represents a single neuronal impulse, and each row of dots represents a single trial. The results of eight trials are shown for each stimulus condition. Bar graphs summarize the mean response for each condition. Note the absence of multisensory enhancement to the combination of unimodal stimuli. In fact, the combined response $(V S)$ is somewhat less than the best unimodal response, a difference that failed to reach statistical significance. Error bars represent SEM, and the dashed line (sum) shows the predicted response on the basis of linear summation. Bottom, Representative oscillographic traces for a single trial of each of the conditions.

visual-somatosensory neuron depicted in Figure 6, which responded to the stimulus combination in a manner that was statistically indistinguishable from its response to either unimodal stimulus. The neuron showed neither a simple linear summation that would be predicted if the two modality-specific inputs were passively summed nor the profound response enhancement that characterizes responses to spatially aligned multisensory cues in the adult (see below). Although the absence of a linear summation of the modality-specific inputs in many of these neurons suggests that active processes are involved in defining their responses, to distinguish these immature multisensory neurons from those of their adult counterparts they were operationally categorized as "nonintegrating." This designation refers only to their inability to exhibit significant multisensory enhancement or depression (see Materials and Methods).

An abrupt developmental change became evident at the end of the fourth postnatal week. Some multisensory neurons now exhibited significant and robust enhancements in their responses when presented with spatially and temporally coincident stimuli from two sensory modalities, as well as pronounced response depression when the stimuli were spatially disparate. Operation- ally, these neurons were categorized as "integrating." Typically, when the modality-specific responses of these neurons were weak, the enhanced responses to these stimulus combinations far exceeded the sum of the two individual responses (Fig. 7) (also see the discussion of "inverse effectiveness" below). Surprisingly, the magnitude of this multisensory enhancement did not increase as the animals matured. In fact, the magnitude of the interactions that were obtained in animals ranging in age from 28-135 dpn was not significantly different from that observed in adults (Fig. 8). Because there were very few of these multisensory integrating neurons in young animals, however, the average enhancement of responses in the SC as a whole was comparatively low. It increased as the proportion of multisensory neurons capable of integration increased, as shown in Figure 8.

What did change with time was the incidence of multisensory neurons that exhibited multisensory integration (Fig. 9). Such neurons appeared abruptly, and their incidence rose rapidly during the ensuing 3-4 weeks. During the next 6-8 weeks, a more gradual rise in their incidence established adult-like levels of integrating neurons (Fig. 9).

Neurons capable of multisensory integration were more mature 

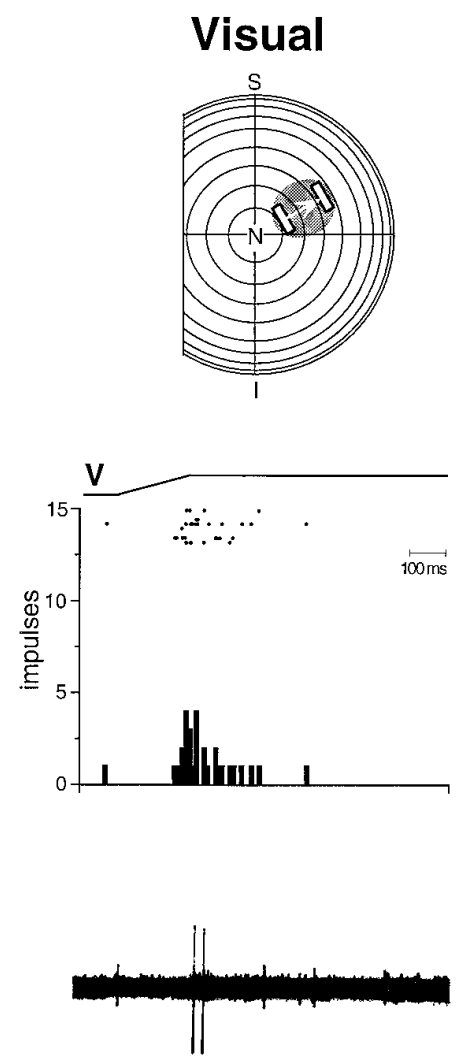

Auditory

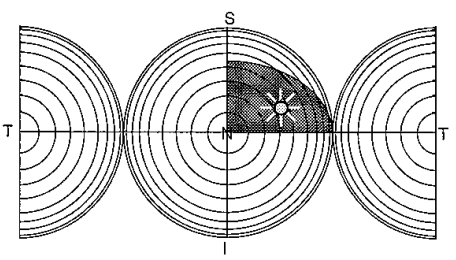

A
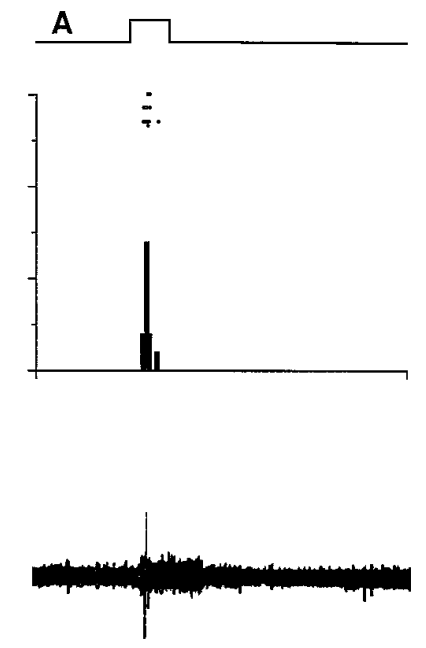

Visual + Auditory
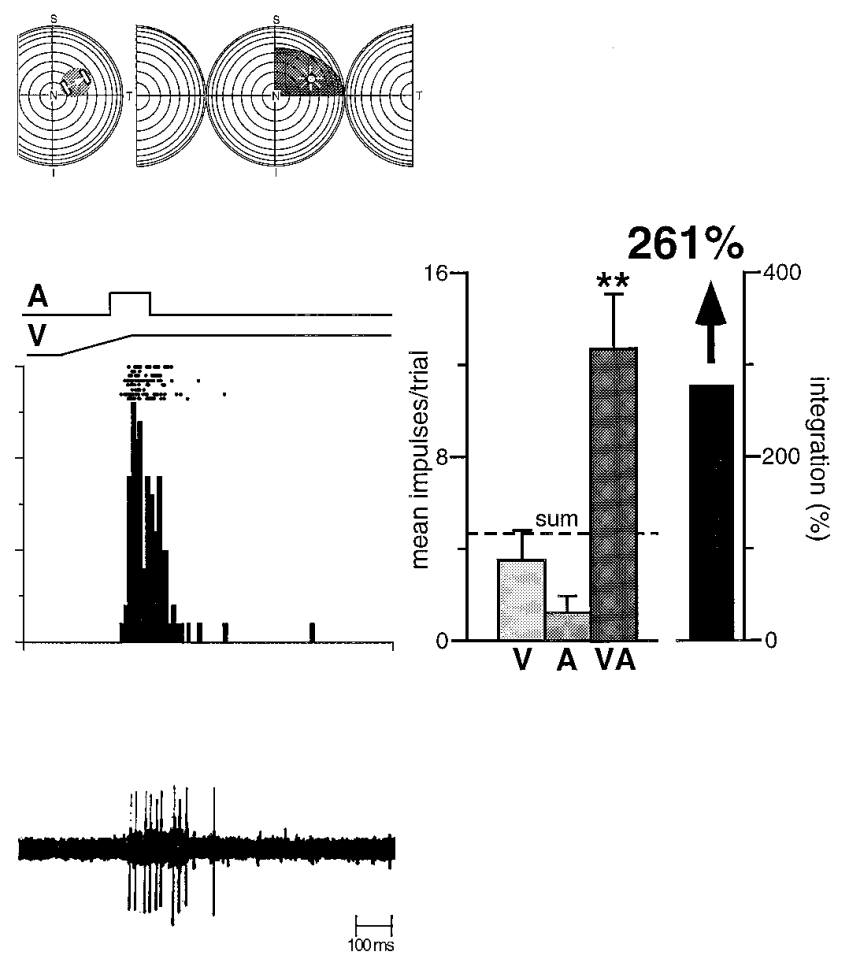

Figure 7. Multisensory neurons exhibiting the capacity to integrate cross-modality cues to significantly enhance (or depress) responses are first seen in the fifth postnatal week and are adult-like in many ways (for conventions, see Figs. 4 and 6). The auditory stimulus is a speaker, the position of which is depicted by the icon within the receptive field. Note the adult-like receptive fields and the significant response enhancement to the combination of the visual and auditory stimuli. ${ }^{* *} p<0.01$.

than age-matched nonintegrating neurons in various response properties. Two representative examples of these contrasting profiles are presented in Figure 10. Both neurons shown are visualauditory, and both were found in the same $35 \mathrm{dpn}$ animal. The responses of the first of these (Fig. 10, top) were not altered by multisensory inputs (i.e., they did not integrate). The visual and auditory receptive fields of this neuron were quite large, and both modality-specific response latencies were significantly longer than in the adult (mean visual latency $=155 \mathrm{msec}$ vs $83 \mathrm{msec}$ in the adult; $p<0.01$; mean auditory latency $=61 \mathrm{msec}$ vs $18 \mathrm{msec}$ in the adult; $p<0.05)$. In contrast, the neuron that exhibited adult-like multisensory integration (Fig. 10, bottom) had receptive fields comparable in size to those of the adult, as well as mature response latencies (visual $=87 \mathrm{msec}$; auditory $=22 \mathrm{msec}$ ).

Receptive field size was found to be an excellent predictor of the ability of a given neuron to exhibit multisensory integration. In neonatal multisensory neurons with adult-sized receptive fields (either visual, auditory, or somatosensory), the probability that the neuron would exhibit multisensory integration was nearly identical to the adult value, as shown in Figure 11. Conversely, in multisensory neurons with immature visual and auditory receptive fields ( $>200 \%$ of the mean adult size), the probability of multisensory integration was $<0.15$ and was zero for neurons with somatosensory receptive fields $>200 \%$ of adult size. Neonatal receptive fields of intermediate size (i.e., between 100 and 200\% of adult values) had intermediate probabilities of exhibiting multisensory integration (Fig. 11).

Even at the earliest stages at which neurons exhibited the capacity to integrate multisensory stimuli, they seemed to be governed by some of the same integrative principles that characterize the adult. This was quite evident in terms of how the spatial relationships among different stimuli dictate the multisensory product, and as long as the stimuli were combined so that both were located within their modality-specific receptive fields, response enhancement resulted (Fig. 12). Conversely, when one of the stimuli was presented outside its receptive field, either no interaction occurred or response depression resulted (Fig. 12).

The influence of temporal disparity on multisensory integration was determined by systematically varying the delay between the onset of two different sensory stimuli. Although the best temporal disparity for optimum response enhancement varied considerably among the neurons studied at each age (from 0 to $100 \mathrm{msec}$ ), maximal interactions were generally $(12 / 17 ; 71 \%)$ achieved when the interval produced an overlap of the peak periods of unimodal discharge. As the interval between the stimuli was lengthened or shortened from this optimum so that the peak unimodal responses were increasingly disparate in time, there was a systematic decline in the magnitude of the response enhancement. The most notable exception to this was seen in the youngest neurons tested for temporal effects (35-42 dpn). In these neurons, multisensory interactions were seen only when stimuli were presented simultaneously (Fig. 13b).

Even young (i.e., 49-70 dpn) neurons exhibiting multisensory integration at disparities of $50 \mathrm{msec}$ or greater still had substantially smaller temporal "windows" than those seen in older animals (Fig. 13). As development progressed, these interactive 


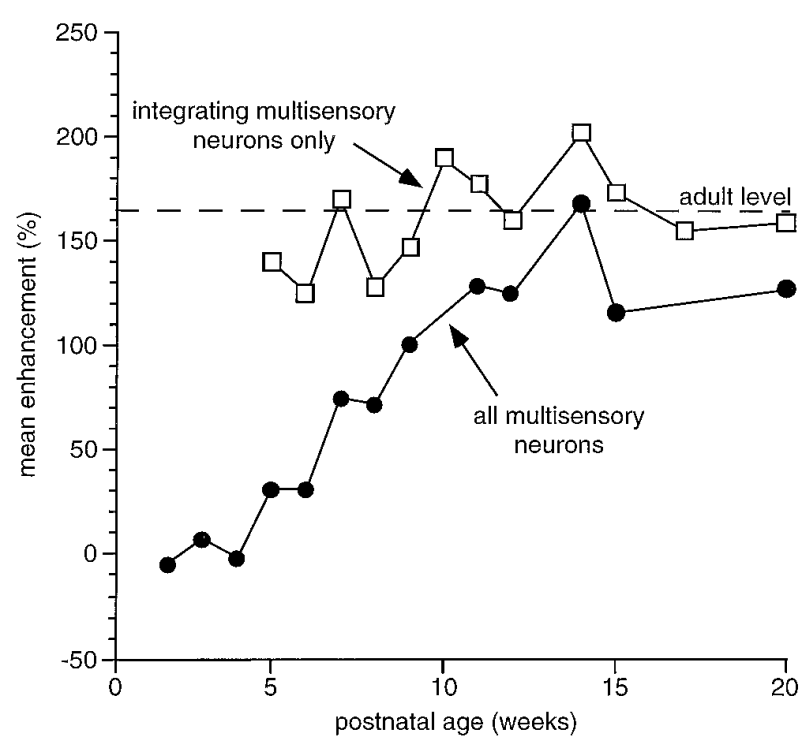

Figure 8. As soon as multisensory neurons develop the capacity to integrate cross-modality cues, the magnitude of the enhancement they exhibit to spatially and temporally coincident stimuli is adult-like. This is evident from the line connecting the open squares. Nonetheless, because the number of integrative neurons matures gradually over time, the population profile takes $\sim 3$ months to mature. Thus, the average enhancement for all multisensory neurons increases gradually as a function of postnatal age as shown by the line connecting the closed circles. The dashed line represents the mean adult level of multisensory enhancement.

windows gradually increased in size (Fig. 13c). In adults, the average temporal window spans $\sim 250 \mathrm{msec}$, and in some neurons significant response enhancements were produced at temporal asynchronies as large as 500-700 msec.

Regardless of age, proportionately greater multisensory enhancements were produced by pairing individual unimodal stimuli whose effectiveness was weakest. This characteristic, defined as "inverse effectiveness" (Meredith and Stein, 1986a), was as evident in young multisensory neurons (i.e., $20 \mathrm{dpn}$ ) as it was in the adult (Fig. 14).

\section{DISCUSSION}

\section{Developmental chronology of sensory responses}

The present results are consistent with previous observations showing that the sensory modalities represented in the deep layers of the SC have a sequential developmental chronology. Somatosensory-responsive neurons are present at birth (and before) (Stein et al., 1973a), and auditory-responsive neurons appear late in the first postnatal week, but visual responses are the most developmentally delayed and do not become evident in the deeper layers until near the end of the third postnatal week. The present results also show that multisensory neurons follow a developmental sequence (auditory-somatosensory neurons appear at $12 \mathrm{dpn}$, followed by visual-multisensory neurons at $20 \mathrm{dpn}$ ) that parallels the chronology of unimodal responses as well as the appearance of overt orientations to modality-specific stimuli (Windell, 1930; Fox, 1970; Norton, 1974; Villablanca and Olmstead, 1979; Sireteanu and Maurer, 1982; Larson and Stein, 1984).

The responses of sensory-responsive SC neurons in very young animals are quite immature. Their most effective stimuli rarely yield $>1-2$ action potentials, their response latencies are substantially longer than are those in the adult, their receptive fields are exceedingly large, and they respond in a nonselective manner to modality-specific stimuli. During development, the gradual shortening of response latencies and the increasing sensory responsiveness presumably are attributable, at least in part, to the myelination of ascending afferent pathways (Tilney and Casamajor, 1924; Windell, 1930; Moore et al., 1976). The decline in receptive field size likely reflects the pruning of exuberant projections and/or the maturation of GABAergic inhibitory circuits within the SC (Mize, 1988, 1992) as well as in structures that project to the SC (Hogan and Berman, 1994) .

The maturation of response selectivity, which is most evident in visually responsive neurons, is likely attributable to the gradual functional onset of specific corticotectal inputs (for review, see Stein, 1984). Unlike the superficial SC layers, which receive substantial retinotectal projections, the predominant visual input to the deeper layers comes from regions of extrastriate cortex (Huerta and Harting, 1984). Most notable among these inputs are those arising in the lateral suprasylvian (LS) and anterior ectosylvian (AES) cortices (Tortelly et al., 1980; Baleydier et al., 1983; Segal and Beckstead, 1984; Berson, 1985). Removal of the LS corticotectal input is particularly effective in degrading or eliminating visual responsiveness in many deep layer neurons (Ogasawara et al., 1984; Hardy and Stein, 1988). This dependence on cortex for general responsiveness seems to be most pronounced for the visual representation in the SC, because removal of auditory and somatosensory corticotectal inputs has substantially less impact on unimodal auditory and somatosensory SC responses (Clemo and Stein, 1986; Meredith and Clemo, 1989; Wallace and Stein, 1994). Thus, it seems that the delayed maturation of visual corticotectal neurons (Stein and Gallagher, 1981) is the most likely explanation for the relative immaturity and protracted development of deep layer SC visual responses.

\section{The appearance of multisensory integration}

Although multisensory neurons first appear toward the end of the second postnatal week, they are comparatively rare at this time, and their incidence increases gradually over a 10-12 week period. More conspicuous than their low numbers at this stage of development, however, is the striking absence of their ability to inte-

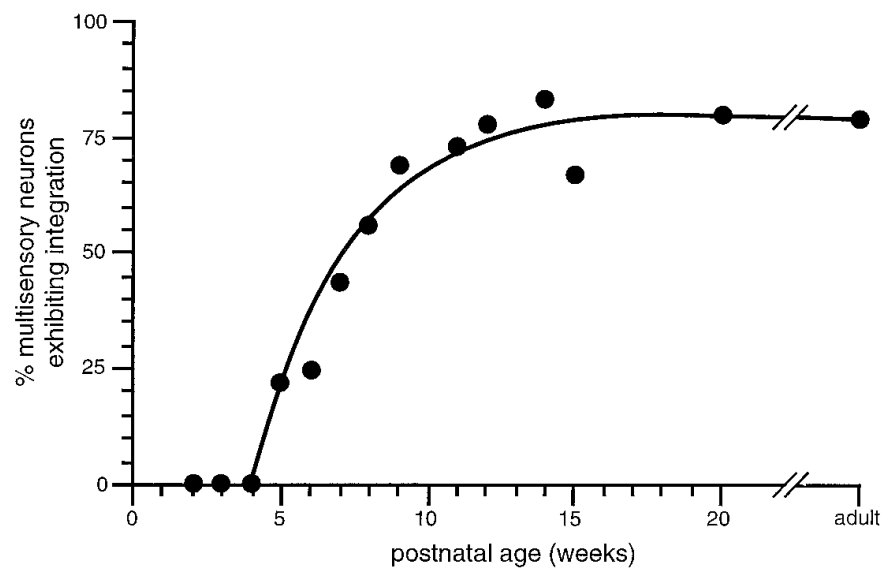

Figure 9. Once initiated, the development of multisensory neurons capable of integrating cross-modality stimuli is rapid. The percentage of the multisensory population exhibiting significant $(p<0.05)$ integration is plotted here as a function of postnatal age. Note the delayed onset of multisensory integration, followed by the rapid rise in the proportion of neurons capable of such integration beginning at 5 weeks and reaching the adult-like proportion at 9-10 weeks. 


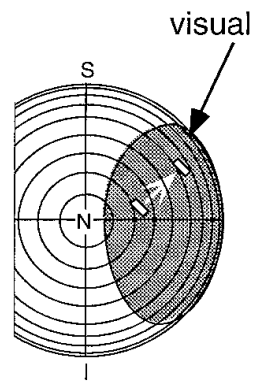

Visual alone

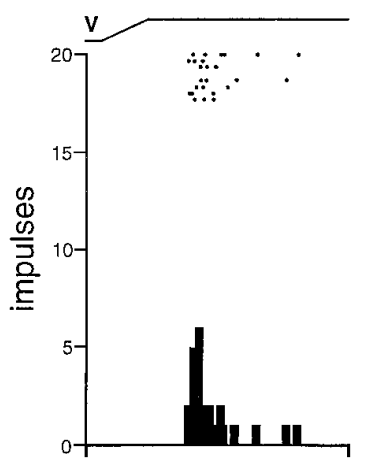

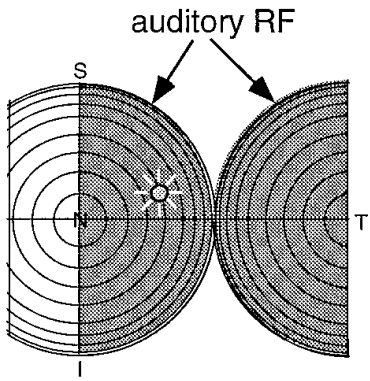

Auditory alone

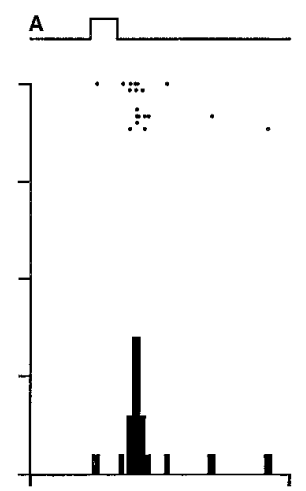

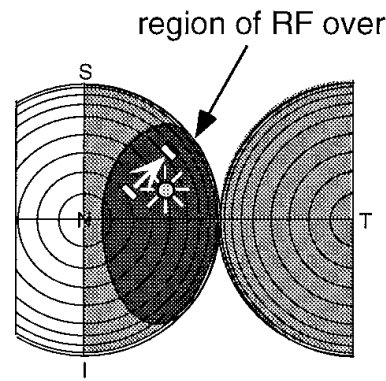

Visual + Auditory

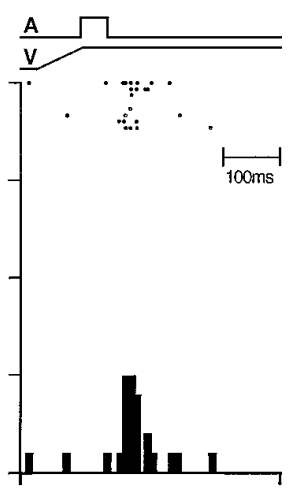

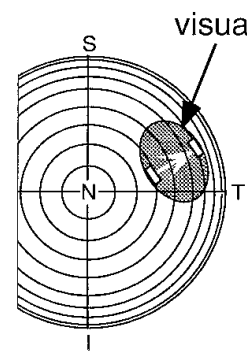

Visual alone

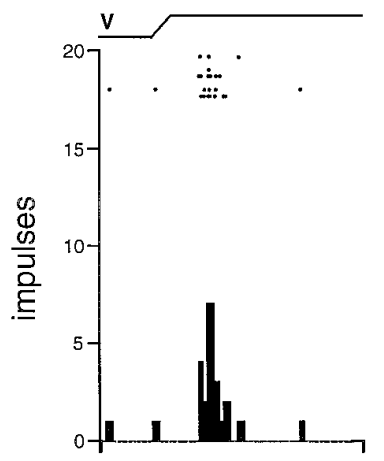

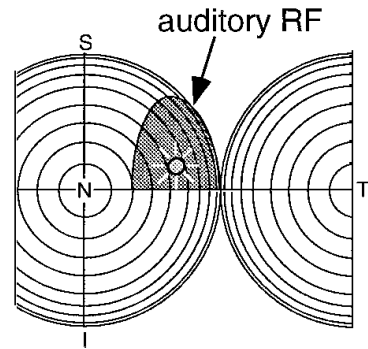

Auditory alone

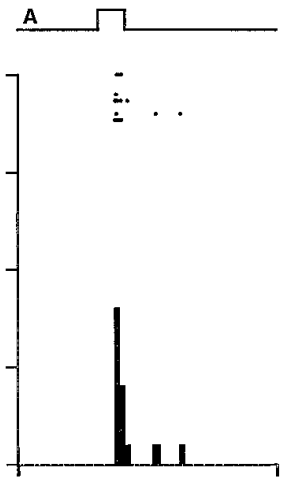

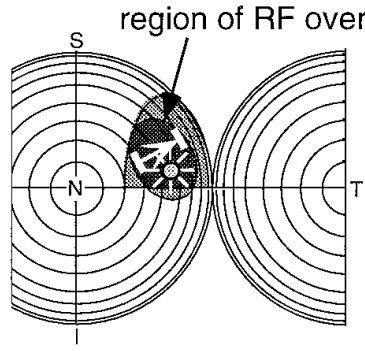

Visual + Auditory

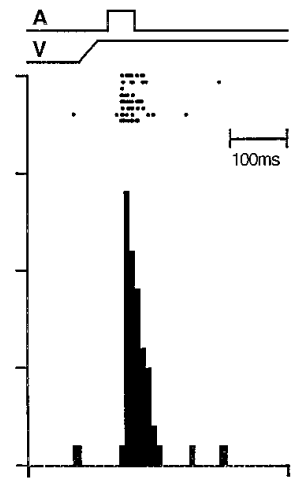

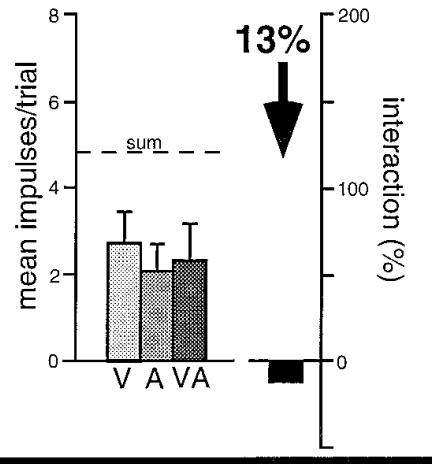

Figure 10. A mixture of multisensory neurons incapable of integrating cross-modality cues (top) and those that had adult-like multisensory integration (bottom) was evident in the same animals. Note the large receptive fields in the "nonintegrating" neuron in this 35 dpn animal, and the adult-like receptive fields in the integrating neuron. ${ }^{*} p<0.05$. See Figures 4 and 6 for conventions. 


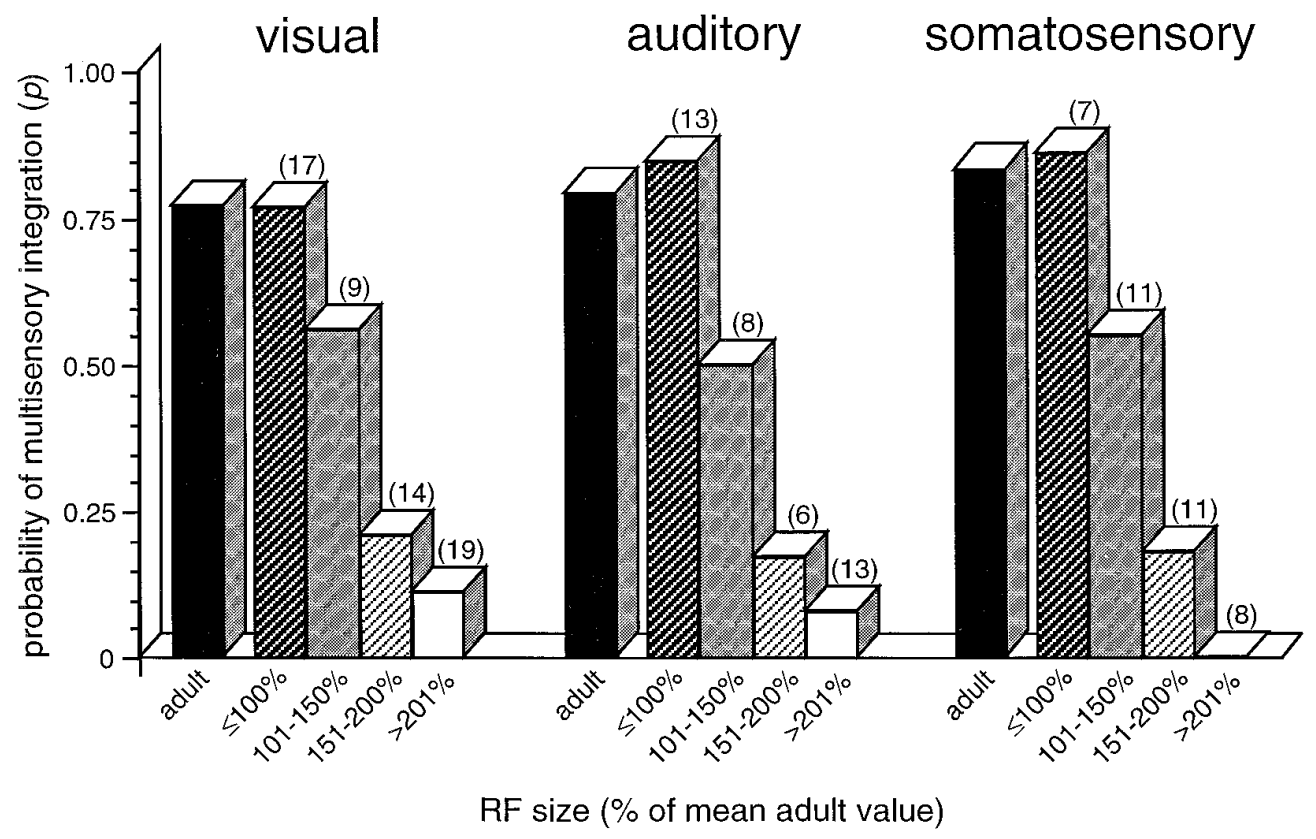

Figure 11. Receptive field size is an excellent predictor of the capacity for multisensory integration. The probability of adult-like multisensory integration in individual neonatal SC neurons is plotted as a function of receptive field size for each of the three modalities. Adult data (black bars) are shown for comparison. Note the high probability of integration for neonatal neurons with adult-size receptive fields and the precipitous decline in integrative probability for neurons with receptive field sizes $>150 \%$ of the adult mean. Because receptive field size varies as a function of position in the SC (rostral receptive fields are smaller than caudal receptive fields), for the purposes of this analysis the SC was divided into four anterior-posterior zones. In this way, the receptive field size of a neonatal neuron was calculated as a percentage of the mean adult receptive field size within the same anterior-posterior zone. Numbers in parentheses show number of neurons in each category.

grate sensory inputs to significantly enhance or degrade the responses evoked by modality-specific stimuli. Many of these first "multisensory" neurons respond to two modality-specific stimuli no differently than they do to the one that is most effective. The observation that this is not the result of a maximal response to the best modality-specific stimulus indicates that some active processes are already operative in limiting the responses of these multisensory neurons.

At about the end of the fourth postnatal week, an abrupt change occurs. Multisensory neurons appear that respond to stimulus combinations with a highly enhanced discharge, typically exceeding that predicted by summing the two unimodal responses. Nonetheless, because the number of such "integrating" neurons increases only gradually with development, it is not until $\sim 3$ postnatal months that the full complement of neurons capable of multisensory integration is present and that the SC can be considered adult-like from a population perspective. On the basis of such findings, it is predicted that multisensory attentive and orientation behaviors (Stein et al., 1989) will show a similar developmental time course.

\section{The contraction of receptive fields and multisensory processes}

At about the time that multisensory integration first appears, a contraction of modality-specific receptive fields is first observed. This finding is interesting in light of current debates on the role of visual experience in the development of the auditory map (Withington-Wray et al., 1990; Knudsen and Brainard, 1991; King and Carlile, 1993; Thornton et al., 1995). If visual signals are "instructing" the developing auditory map (and possibly the developing somatosensory map as well), one would predict that visual receptive fields would contract before the contraction of nonvisual receptive fields. Such a finding would be most apparent, and most compelling, in the same multisensory neuron where the mismatch in visual-nonvisual receptive field sizes could be readily assessed. There was no evidence for such a mismatch in the current study. Receptive field restriction seemed to occur concomitantly across modalities (in a given multisensory neuron). Nevertheless, it remains possible that the interval between visual receptive field contraction and the induced contraction of its auditory (or somatosensory) receptive field is so short that the effect would have not been apparent here.

Receptive field contraction proved to be an excellent predictor of the ability of a given neuron to engage in multisensory integration. At any given developmental stage, neurons incapable of multisensory integration had large receptive fields, whereas their integrating counterparts had receptive fields that closely resembled those seen in the adult. The strong correlation between these two events suggests that they may be driven by the same underlying developmental process or processes. One candidate molecule that has been linked to both of these events is the NMDA receptor. This receptor plays an important role in map formation (and, by extension, in receptive field consolidation) (Scherer and Udin, 1989; Cline and Constantine-Paton, 1990; Schnupp et al., 1995) and has been implicated in the development (Kao et al., 1994a) and maintenance of normal SC sensory processing (Binns and Salt, 1996; Graham et al., 1996).

\section{The adult-like nature of early multisensory integration}

Surprisingly, even the earliest neurons that exhibit multisensory integration resemble their adult counterparts in terms of the magnitude of the response enhancements evoked and their adherence to the spatial, inverse effectiveness, superadditive, and receptive field preservation principles on which this integration is believed to be based (Meredith and Stein, 1986a,b). Thus, when two different sensory stimuli are presented within their respective 


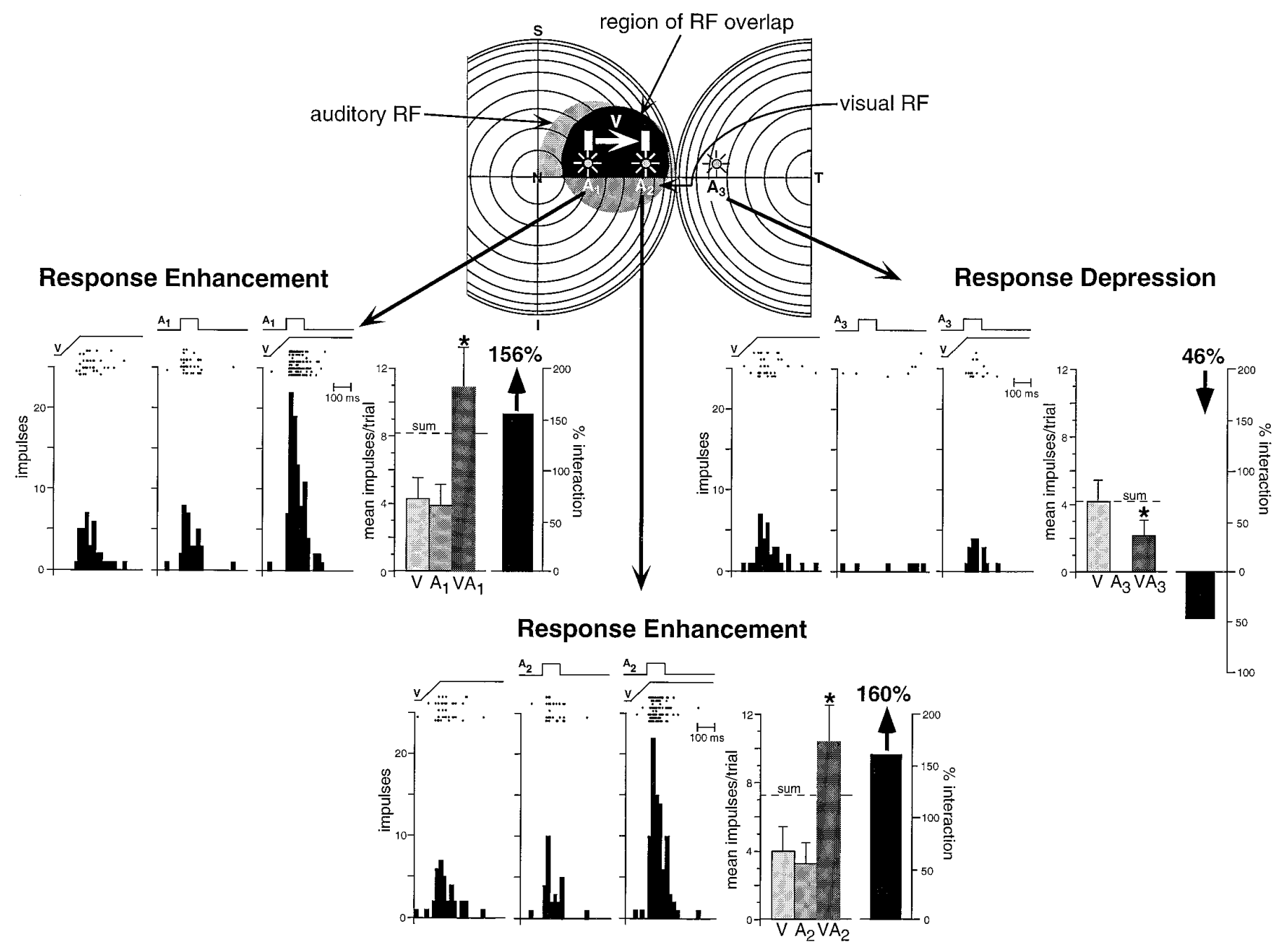

Figure 12. The spatial principle of multisensory integration was seen as soon as neurons developed the capacity to integrate cross-modality cues. This is illustrated in a $35 \mathrm{dpn}$ visual-auditory neuron. At the top are shown the receptive fields of this neuron, with the region of receptive field overlap depicted in black. In this paradigm, the visual stimulus was a bar of light moving in the direction of the arrow, while the auditory stimulus was presented at three different locations $\left(A_{1}, A_{2}\right.$, and $\left.A_{3}\right)$. When both stimuli were presented within their respective receptive fields (middle left and bottom), their combination resulted in a significant response enhancement. When the auditory stimulus was presented outside its receptive field (middle right), the visual-auditory stimulus combination produced significant response depression. ${ }^{*} p<0.05$.

receptive fields, response enhancement is typically the result, but when one of the stimuli is presented outside its receptive field, either response depression or no interaction results. Although no attempt was made to quantitatively compare spatial disparity/ interaction functions in neonates and adults, the general spatial principle of multisensory integration (Meredith and Stein, 1996) seems to be applicable in even the youngest neurons capable of multisensory integration. Similarly, in both neonatal and adult neurons, the least effective unimodal stimuli gave rise to the largest proportionate response enhancements (inverse effectiveness principle) and produced enhancements that far exceeded those predicted on the basis of a simple linear summation of the two unimodal responses (superadditivity principle). As in the adult, no examples were found in which the unimodal receptive field properties of a neuron were altered as a result of multisensory integration (principle of preservation of receptive field properties).

Nonetheless, several interesting differences were noted between neonates and adults in terms of how the temporal relationship between the stimuli affected multisensory integration. In the vast majority of adult multisensory neurons, integration can take place even if the two modality-specific stimuli are separated by a significant temporal interval. Generally, this temporal "window" is on the order of $250 \mathrm{msec}$ and often can be substantially longer (Meredith and Stein, 1986a); however, in the earliest neurons that exhibited multisensory integration, such a temporal window was absent. Multisensory interactions were generated exclusively at simultaneity and were eliminated if the stimuli were displaced from one another by as little as $50 \mathrm{msec}$. In the adult, overlapping the peak periods of unimodal discharge results in maximal multisensory interactions (the temporal principle of multisensory integration) (Meredith et al., 1987), whereas simultaneous stimulus presentation in the neonate failed to overlap these peak periods yet still was the only temporal interval to give rise to an interaction. With development, temporal windows gradually appeared and increased in size, and the temporal principle came to characterize most multisensory interactions.

Although the bases for these temporal differences are not yet understood, they may relate to the very different behavioral rep- 

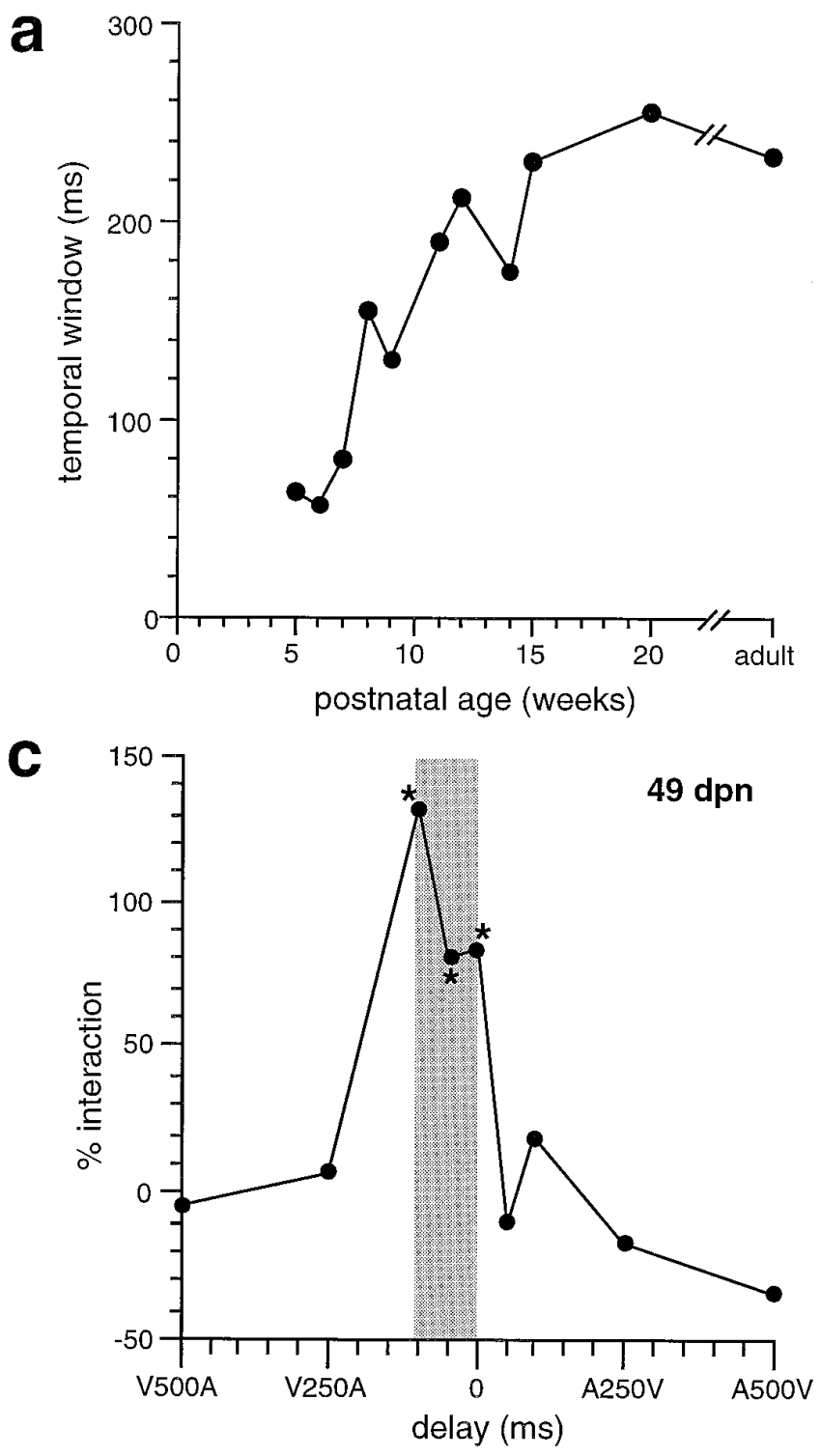
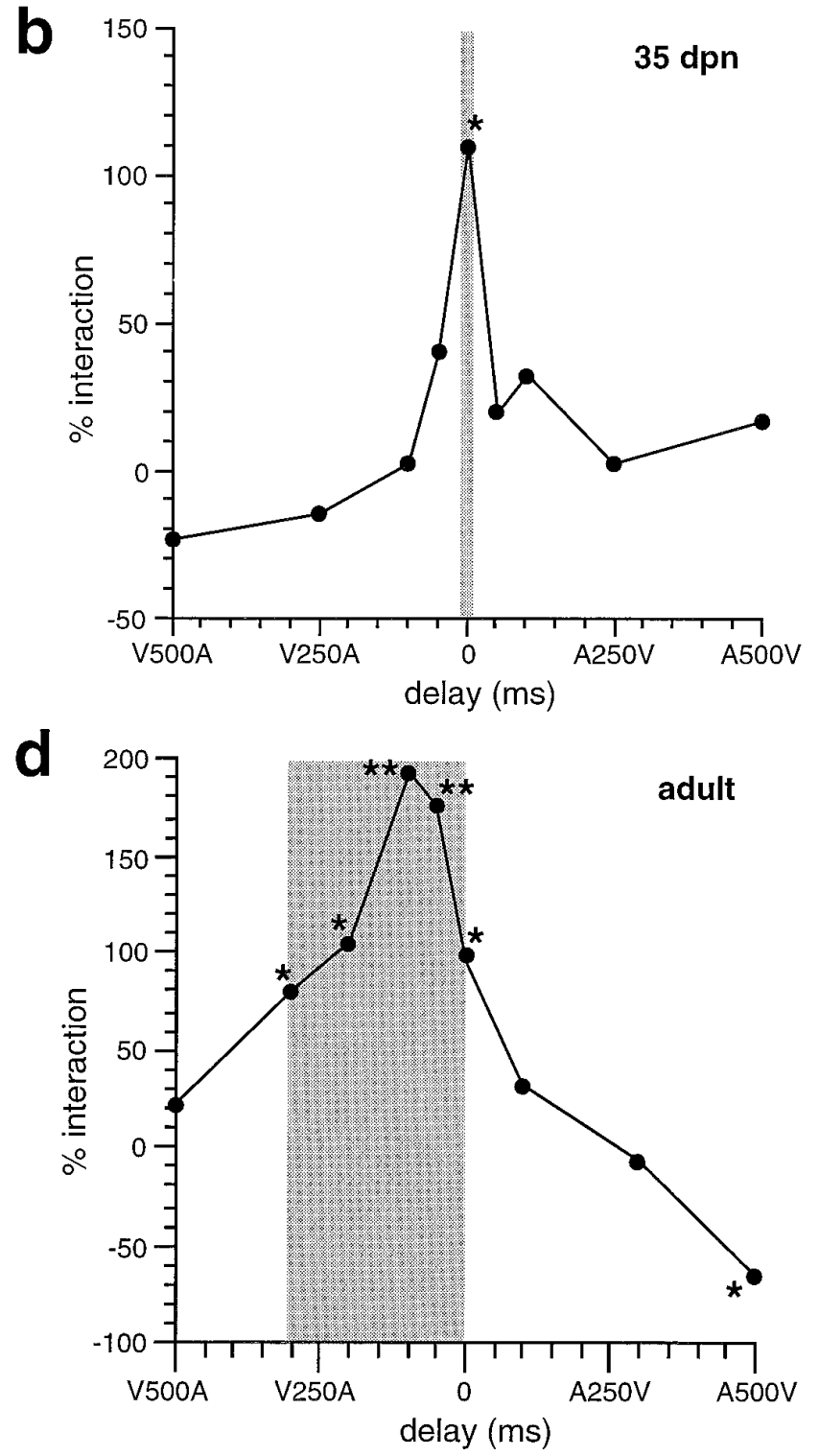

Figure 13. The temporal window in which multisensory integration takes place increases during development. $a$, The size of the temporal window is plotted as a function of postnatal age. $b$. Integration as a function of temporal delay in a $35 \mathrm{dpn}$ visual-auditory neuron. $V 500 A$ represents the visual stimulus preceding the auditory stimulus by $500 \mathrm{msec}, A 500 \mathrm{~V}$ represents the converse, and 0 represents the simultaneous presentation of the two stimuli. The shading shows the temporal window within which statistically significant interactions $(* p<0.05 ; * *<0.01)$ were generated. Note that in this case interactions were generated only at simultaneity. $c$, A similar plot for a 49 dpn visual-auditory neuron. Note the wider (100 msec) temporal window. $d$, A typical plot for an adult visual-auditory neuron. Note the wide $(300 \mathrm{msec})$ temporal window and the significant response depression observed when the auditory stimulus preceded the visual stimulus by $500 \mathrm{msec}(A 500 \mathrm{~V})$.

ertoires of neonates and adults. During the first several postnatal weeks the kitten's sensory world is centered around events occurring in close spatial proximity (e.g., stimuli derived from littermates and the mother). As a result of this, virtually all sensory stimuli impinge on their respective receptor epithelia simultaneously. As the animal's sensory and sensorimotor world expands so that it must deal with increasingly distant events (e.g., hunting, exploring), there is an expansion of the temporal window during which multiple sensory cues can interact. An investigation in which both behavioral and physiological responses are determined in the same animals would be helpful in evaluating this possibility.

\section{A presumptive role of cortex in the appearance of multisensory integration}

As noted above, there is a maturational lag between the appearance of multisensory neurons and the onset of their ability to integrate cross-modality stimuli. Several lines of evidence suggest that this delay may be the result of the late maturation of specific cortical inputs.

In adults, it has recently been shown that deactivation of a region of association cortex, the AES, eliminates multisensory integration in a majority of SC neurons and does so with only minimal effect on their unimodal responses (Wallace and Stein, 1994). These neurons now look very much like those seen in neonates: they are responsive 


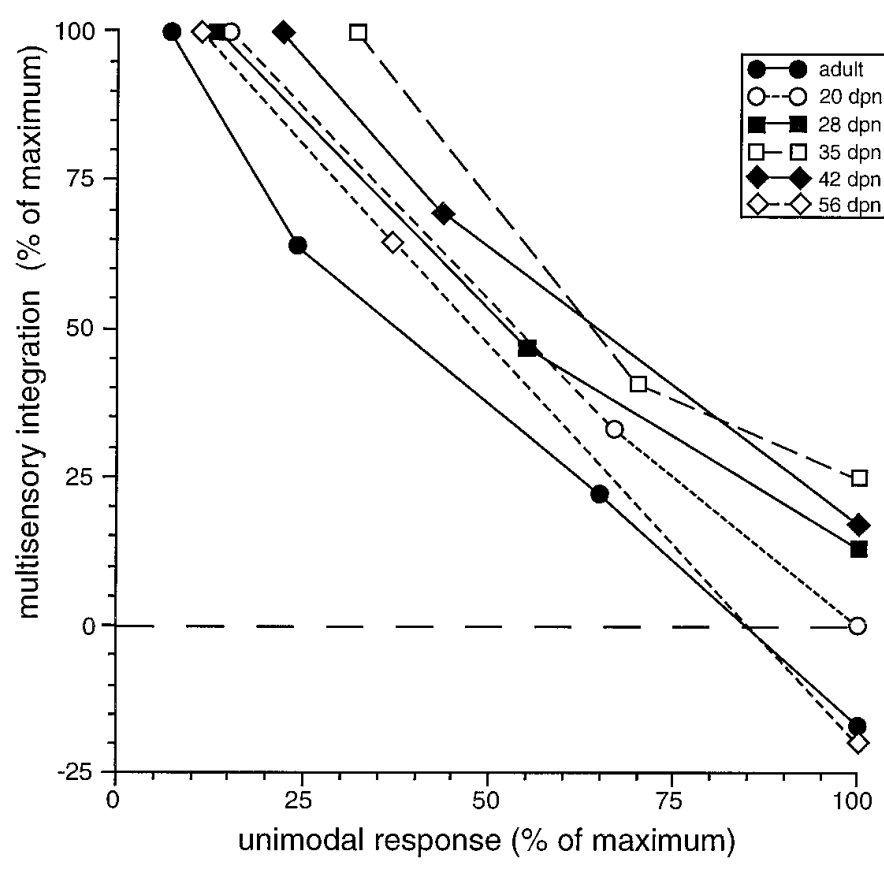

Figure 14. The inverse effectiveness principle of multisensory integration was apparent in multisensory neurons as soon as they developed the capacity to integrate cross-modality cues. The six examples illustrated here, from single multisensory neurons in animals ranging in age from 20 dpn to the adult, showed a very similar relationship between the unimodal response and the integrative product: as the unimodal stimulus became more effective, the level of multisensory integration declined.

to stimuli from more than a single modality but cannot synthesize these inputs to significantly enhance or degrade their modalityspecific responses. On the basis of this, it seems likely that early multisensory neurons lack functional AES corticotectal connections. Presumably, once these inputs become functional, they are able to confer nearly adult-like integrative capabilities on their multisensory SC targets. One possibility is that the final causal event is the development of functional AES corticotectal synapses on multisensory SC neurons.

Yet, whatever final step is necessary to initiate multisensory integration in these SC neurons, it may represent a biological event that is conserved across the various corticotectal pathways, for there is a similarly abrupt onset of corticotectal control over visual SC neurons. In this case it is essential for the appearance of direction selectivity (Stein and Gallagher, 1981). Whether the abrupt developmental onset of corticotectal control is a property unique to sensory projections or one that extends to all corticotectal systems remains to be determined.

\section{REFERENCES}

Baleydier C, Kahungu M, Mauguiere F (1983) A crossed corticotectal projection from the lateral suprasylvian area in the cat. J Comp Neurol 214:344-351.

Berson DM (1985) Cat lateral suprasylvian cortex: Y-cell inputs and corticotectal projections. J Neurophysiol 53:544-556.

Binns KE, Salt TE (1996) Importance of NMDA receptors for multimodal integration in the deep layers of the cat superior colliculus. J Neurophysiol 75:920-930.

Clemo HR, Stein BE (1986) Effects of cooling somatosensory cortex on response properties of tactile cells in superior colliculus. J Neurophysiol $55: 1352-1368$.

Cline HT, Constantine-Paton M (1990) NMDA receptor agonists and antagonists alter retinal ganglion cell arbor structure in the developing frog retinotectal projection. J Neurosci 10:1197-1216.
Fox MW (1970) Reflex development and behavioural organization. In: Developmental neurobiology (Himwich WA, ed), pp 553-580. Springfield, IL: Thomas.

Goldberg JM, Brown PB (1969) Responses of binaural neurons of dog superior olivary complex to dichotic tonal stimuli: some physiological mechanisms of sound localization. J Neurophysiol 32:613-636.

Graham JH, Vaughan JW, Schaafsma SJ, Wallace MT, Stein BE (1996) Role of NMDA receptors in multisensory integration in the superior colliculus. Soc Neurosci Abstr 22:637.

Hardy SC, Stein BE (1988) Small lateral suprasylvian cortex lesions produce visual neglect and decreased visual activity in the superior colliculus. J Comp Neurol 273:527-542.

Hogan D, Berman NE (1994) The development of parvalbumin and calbindin-D28k immunoreactive interneurons in kitten visual cortical areas. Dev Brain Res 77:1-21.

Huerta MF, Harting JK (1984) The mammalian superior colliculus: studies of its morphology and connections. In: Comparative neurology of the optic tectum (Vanegas H, ed), pp 687-773. New York: Plenum.

Kao CQ, Stein BE, Coulter DA (1994a) Postnatal development of excitatory synaptic function in deep layers of superior colliculus. Soc Neurosci Abstr 20:1186.

Kao CQ, McHaffie JG, Meredith MA, Stein BE (1994b) Functional development of a central visual map in cat. J Neurophysiol 72:266-272.

King AJ, Carlile S (1993) Changes induced in the representation of auditory space in the superior colliculus by rearing ferrets with binocular eyelid suture. Exp Brain Res 94:444-455.

Knudsen EI, Brainard MS (1991) Visual instruction of the neural map of auditory space in the developing optic tectum. Science 253:85-87.

Larson M, Stein BE (1984) The use of tactile and olfactory cues in neonatal orientation and localization of the nipple. Dev Psychobiol 17:423-436.

Levine MS, Hull CD, Buchwald NA (1980) Development of motor activity in kittens. Dev Psychobiol 13:357-371.

Meredith MA, Clemo HR (1989) Auditory cortical projection from the anterior ectosylvian sulcus (Field AES) to the superior colliculus in the cat: an anatomical and electrophysiological study. J Comp Neurol 289:687-707.

Meredith MA, Stein BE (1986a) Visual, auditory, and somatosensory convergence on cells in superior colliculus results in multisensory integration. J Neurophysiol 56:640-662.

Meredith MA, Stein BE (1986b) Spatial factors determine the activity of multisensory neurons in cat superior colliculus. Brain Res 365:350-354.

Meredith MA, Stein BE (1996) Spatial determinants of multisensory integration in cat superior colliculus. J Neurophysiol 75:1843-1857.

Meredith MA, Nemitz JW, Stein BE (1987) Determinants of multisensory integration in superior colliculus neurons. 1. Temporal factors. J Neurosci 7:3215-3229.

Meredith MA, Wallace MT, Stein BE (1992) Visual, auditory and somatosensory convergence in output neurons of the cat superior colliculus: multisensory properties of the tecto-reticulo-spinal projection. Exp Brain Res 88:181-186.

Mize RR (1988) Immunocytochemical localization of gamma-aminobutyric acid (GABA) in the cat superior colliculus. J Comp Neurol 276:169-187.

Mize RR (1992) The organization of GABAergic neurons in the mammalian superior colliculus. Prog Brain Res 90:219-248.

Moore CL, Kalil R, Richards W (1976) Development of myelination in optic tract of the cat. J Comp Neurol 165:125-136.

Norton TT (1974) Receptive-field properties of superior colliculus cells and development of visual behavior in kittens. J Neurophysiol 37:674-690.

Ogasawara K, McHaffie JG, Stein BE (1984) Two visual corticotectal systems in cat. J Neurophysiol 52:1226-1245.

Scherer WJ, Udin SB (1989) $N$-methyl-D-aspartate antagonists prevent interaction of binocular maps in Xenopus tectum. J Neurosci 9:3837-3843.

Schnupp JWH, King AJ, Smith AL, Thompson ID (1995) NMDAreceptor antagonists disrupt the formation of the auditory space map in the mammalian superior colliculus. J Neurosci 15:1516-1531.

Segal RL, Beckstead RM (1984) The lateral suprasylvian corticotectal projection in cats. J Comp Neurol 225:259-275.

Sireteanu R, Maurer D (1982) The development of the kitten's visual field. Vision Res 22:1105-1111.

Stein BE (1984) Development of the superior colliculus. Annu Rev Neurosci 7:95-125.

Stein BE, Gallagher H (1981) Maturation of cortical control over superior colliculus neurons in cat. Brain Res 223:429-435. 
Stein BE, Meredith MA (1993) The merging of the senses. New York: MIT.

Stein BE, Labos E, Kruger L (1973a) Sequence of changes in properties of neurons of superior colliculus of the kitten during maturation. J Neurophysiol 36:667-679.

Stein BE, Labos E, Kruger L (1973b) Determinants of response latency in neurons of the superior colliculus in kittens. J Neurophysiol 36:680-689.

Stein BE, Magalhaes-Castro B, Kruger L (1976) Relationship between visual and tactile representations in cat superior colliculus. J Neurophysiol 39:401-419.

Stein BE, Clamann HP, Goldberg SJ (1980) Superior colliculus: control of eye movements in neonatal kittens. Science 210:78-80.

Stein BE, Meredith MA, Huneycutt WS, McDade L (1989) Behavioral indices of multisensory integration: orientation to visual cues is affected by auditory stimuli. J Cognit Neurosci 1:12-24.

Stein BE, Meredith MA, Wallace MT (1993) Development and neural basis of multisensory integration. In: The development of intersensory perception: comparative perspectives (Lewkowitz DJ, Lickliter R, eds), pp 81-105. Hillsdale, NJ: Erlbaum.

Thornton SK, Ingham NJ, Withington DJ (1995) Visual movement and pattern are important for the development of a map of auditory space in the guinea pig superior colliculus. Exp Brain Res 106:257-264.

Tilney F, Casamajor L (1924) Myelinogeny as applied to the study of behavior. Arch Neurol Psychiatry 12:1-66.

Tortelly A, Reinoso-Suarez F, Llamas A (1980) Projections from non- visual cortical areas to the superior colliculus demonstrated by retrograde transport of HRP in the cat. Brain Res 188:543-549.

Van Hof-Van Duin J (1976) Development of visuomotor behaviour in normal and dark-reared cats. Brain Res 104:233-241.

Villablanca JR, Olmstead CE (1979) Neurological development in kittens. Dev Psychobiol 12:101-127.

Wallace MT, Stein BE (1994) Cross-modal synthesis in the midbrain depends on input from cortex. J Neurophysiol 71:429-432.

Wallace MT, Meredith MA, Stein BE (1993a) Converging influences from visual, auditory, and somatosensory cortices onto output neurons of the superior colliculus. J Neurophysiol 69:1797-1809.

Wallace MT, Meredith MA, Stein BE (1993b) Development of multisensory integration in cat superior colliculus. Soc Neurosci Abstr 19:240.

Wallace MT, Wilkinson LK, Stein BE (1996) Representation and integration of multiple sensory inputs in primate superior colliculus. J Neurophysiol 76:1246-1266.

Wilkinson LK, Meredith MA, Stein BE (1996) The role of anterior ectosylvian cortex in cross-modality orientation and approach behavior. Exp Brain Res 112:1-10.

Windell WF (1930) Normal behavioral reactions of kittens correlated with the postnatal development of nerve-fiber density in the spinal gray matter. J Comp Neurol 50:479-503.

Withington-Wray DJ, Binns KE, Keating MJ (1990) The maturation of the superior collicular map of auditory space in the guinea pig is disrupted by developmental visual deprivation. Eur J Neurosci 2:682-692. 